MATHEMATICS OF COMPUTATION

Volume 75 , Number 253 , Pages $113-150$

S 0025-5718(05)01770-9

Article electronically published on August 12, 2005

\title{
ERROR ESTIMATE FOR THE APPROXIMATION OF NONLINEAR CONSERVATION LAWS ON BOUNDED DOMAINS BY THE FINITE VOLUME METHOD
}

\author{
MARIO OHLBERGER AND JULIEN VOVELLE
}

\begin{abstract}
In this paper we derive a priori and a posteriori error estimates for cell centered finite volume approximations of nonlinear conservation laws on polygonal bounded domains. Numerical experiments show the applicability of the a posteriori result for the derivation of local adaptive solution strategies.
\end{abstract}

\section{INTRODUCTION}

Let $\Omega$ be an open convex polygonal bounded domain in $\mathbb{R}^{d}, d=2,3$, endowed with the Euclidean norm $|\cdot|$, and let $T \in \mathbb{R}^{+}$. We consider the following initial boundary value problem for nonlinear scalar conservation laws:

$$
\begin{aligned}
c_{t}+\nabla \cdot \mathbf{F}(x, t, c) & =0 \text { in } \Omega \times(0, T), \\
c(\cdot, 0) & =c_{0} \text { in } \Omega, \\
c(x, t) & =\bar{c}(t, x) \text { in } \partial \Omega \times(0, T) .
\end{aligned}
$$

The flux in equation (11) is given by the function $\mathbf{F} \in \mathcal{C}^{1}\left(\Omega \times(0, T) \times \mathbb{R} ; \mathbb{R}^{d}\right)$; the functions $c_{0} \in L^{\infty}(\Omega)$ and $\bar{c} \in L^{\infty}(\partial \Omega \times(0, T))$ are the initial and boundary data of the problem (1)-(3), respectively.

The finite volume methods are known to be well suited for the discretization of conservation laws. A basic account for this claim is the fact that, by construction, they respect the conservation principle which constitutes the root of equation (1). Indeed, the evolution of the discrete unknown $c_{K}$ in each control volume $K$ is given by the equation

$$
|K| c_{K}^{n+1}=|K| c_{K}^{n}+\Delta t^{n} \sum_{\sigma \in \partial K} Q_{\sigma}^{n}
$$

in which we denote abusively by $\partial K$ the set of faces of $K$ and where $|K|$ is the volume of $K$. Equation (4) is the expression of the fact that the discrete evolution of $c_{K}$ is governed by the values of the discrete fluxes $Q_{\sigma}^{n}$ across the boundary of $K$ in the time interval $\left[t^{n}, t^{n+1}\right]$. It is the choice of these numerical fluxes $Q_{\sigma}^{n}$ that determine the finite volume method. In what follows, we will specifically consider three point finite volume schemes with monotone fluxes (see (13)-(17)).

Received by the editor January 12, 2004 and, in revised form, September 9, 2004.

2000 Mathematics Subject Classification. Primary 35L65, 65N15.

Key words and phrases. Hyperbolic equation, initial boundary value problem, finite volume method, error estimate.

(C)2005 American Mathematical Society 
This category of schemes encloses all relevant first order three point finite volume schemes.

Since both (11) and (44) are evolution equations, the main features of the analysis of conservation laws and of their approximations by the finite volume method already appear in the context of the Cauchy problem, i.e., $\Omega=\mathbb{R}^{d}$, and no boundary conditions have to be taken into account. The order of accuracy of the finite volume method for the Cauchy problem is one of these well known features: the first given a priori error estimate is the (sharp) $h^{1 / 2}$ ( $h$ being the size of the mesh) estimate of Kuznetsov [Kuz76] in the 1D case. This estimate remains valid on structured meshes in $\mathbb{R}^{d}$ while, for finite volume schemes on unstructured meshes, the lack of an uniform $B V$ estimate on the numerical solution leads to an error estimate of reduced order $h^{1 / 4}$ KNR95, BCV95, CCL95a, EGH00. Still, in the context of the Cauchy problem, refined error estimates have been given (and their sharpness analyzed), according to the genuine nonlinearity of the flux, to the structure of the entropy solution to (11)-(2), or to the nature of the waves in the solution. We refer to the discussion and compilation made by T. Tang on that profuse subject [Tan01].

For practical applications, a posteriori error estimates are even more important than just convergence rates. Such estimates allow us to extract error indicator information that can be used in order to derive efficient self-adaptive strategies for the finite volume schemes. A posteriori error estimates for finite volume approximations to the Cauchy problem were first derived by Tadmor Tad91 in one space dimension, and by Cockburn and Gau CG95 in the multi-dimensional case. A localized estimate for general flux functions and the derivation of self-adaptive schemes was given in $\mathrm{KO} 00$. Further results for finite volume approximations to the Cauchy problem were obtained in GM00, KO03, KKP02, while finite element approximations to the Cauchy problem were studied in JS95, SH95, HMSW99, HH02. We emphasize that up to now no a posteriori results are available for approximations of the initial boundary value problem (1)-(3).

Although the study of the finite volume method applied to the Cauchy problem (11), (2) has led to the understanding of most of the mechanisms which govern the accuracy of this numerical method of approximation, the initial boundary value problem (11)-(3) has its own interest (for the simple and major reason that the domains under consideration in practical applications can be bounded domains), and its approximation by finite volume schemes deserves an analysis. With that purpose in mind, notice that a new and characteristic feature of the approximation of the initial boundary value problem (11) -(3) by a finite volume scheme is the possible creation of a numerical boundary layer. This numerical boundary layer is a subproduct of the numerical diffusion effects induced by the scheme. Of course, its presence is also related to the way in which the boundary data are implemented in the scheme. Let us specify this point. We consider here and in the following the implementation of boundary data via "ghost control volumes". This is a way to compute the numerical fluxes at the boundary of the domain inspired by the design of the fluxes inside the domain. Indeed, if $\sigma$ is an edge of a control volume $K$ but also one of the edges of the control volume $L$, then the numerical flux $Q_{\sigma}^{n}$ (cf. (4) ) is given as a function of the discrete unknowns $c_{K}^{n}$ and $c_{L}^{n}$ by the formula $Q_{\sigma}^{n}=G_{\sigma}^{n}\left(c_{K}^{n}, c_{L}^{n}\right)$ where, among other properties, the function $G_{\sigma}^{n}$ is nondecreasing with respect to its first argument and nonincreasing with respect to the second. If $\sigma$, edge of a control volume $K$, is now located at the boundary of the domain $\Omega$, 
then a ghost control volume $L$ such that $L \subset \mathbb{R}^{d} \backslash \Omega$ and $\sigma=\bar{K} \cap \bar{L}$ is introduced and the computation of the numerical flux at the boundary $Q_{\sigma}^{n}$ relies on a numerical flux function $G_{\sigma}^{n}$ (nondecreasing with respect to its first argument and nonincreasing with respect to the second) via the formula $Q_{\sigma}^{n}=G_{\sigma}^{n}\left(c_{K}^{n}, c_{L}^{n}\right)$ where the value $c_{L}^{n}$ is a discretization (typically the mean value) of the boundary datum $\bar{c}$ on $\left[t^{n}, t^{n+1}\right) \times \sigma$. This method of computation of the numerical fluxes at the boundary of the domain is classical and ensures the convergence of the finite volume scheme to the entropy solution of the problem (1)-(3) Sze91, BCV95, CCL95a, Vov02. Let us also stress that the proposed finite volume discretization is of rather great importance for practical applications (see the discussion on the implementation of numerical boundary conditions in the approximation of two phase flow problems in EGV03). Before coming back to our considerations of numerical boundary layers and of their influence on the speed of convergence of the finite volume method, let us observe that when systems of conservation laws are considered, the computation of the numerical fluxes at the boundary of the domain by the method of ghost control volumes may be not accurate. Other methods, like reflecting, or absorbing boundary conditions are in use, and, when used, the method of ghost control volumes is associated to the Godunov method for the computation of the flux. In this context, the Godunov method is indeed considered to give the reliable choice of numerical flux functions at the boundary.

The study of the numerical boundary layer has been performed by C. ChainaisHillairet and E. Grenier CHG01, in the 1D case and for modified Lax-Friedrichs schemes on cartesian grids in the multi-dimensional case. Such an analysis gives a precise description of the numerical solution and, as a consequence, the speed of convergence of this solution to the entropy solution of the problem (11)-(3). In the noncharacteristic case with smooth exact solutions, this speed of convergence is proved to be of order $h$ in the $L^{\infty}\left(0, T ; L^{1}(\Omega)\right)$ norm, where $h$ is the size of the mesh.

Unfortunately, the techniques of numerical boundary layer analysis seem difficult to be set when no selected direction of (discrete) derivation exists, as is the case when finite volume schemes on unstructured meshes are used. For such schemes one can therefore think to adapt the technique developed by Kuznetsov Kuz76 for the analysis of the Cauchy problem in the framework of the initial boundary value problem to get error estimates, with the drawback that this tool is not accurate at all to take into account the special phenomena at the boundary of the domain. In the specific situation $\mathbf{F}(x, t, c)=\mathbf{u}(x, t) f(c)$ with $f$ monotone, this drawback can be overcome, for the reason that the inflow and outflow parts of the boundary are determined a priori by the given velocity field $\mathbf{u}$. In [Vig97, Vignal gives an a priori error estimate of order $h^{1 / 4}$ for the initial boundary value problem. However, to our knowledge, for general fluxes $\mathbf{F}$, and general schemes on possibly unstructured meshes, no results or techniques of error estimates which account for the influence of the boundary condition have been delivered. In order to fill in this gap, we adapt the technique of Kuznetsov Kuz76 to the proof of uniqueness of the entropy solution given by F. Otto Ott96, MNRR96, and prove that the error can be estimated by an a posteriori error bound which is at least of order $h^{1 / 6}$ for meshes with mesh size $h$ (see Propositions 5.1 and 5.2). The order, $h^{1 / 6}$, of our a priori error estimate also has to be discussed. Our comments are postponed to Remark [5.9. 
Since the finite volume methods introduce some numerical diffusion effects in the approximation of the entropy solution to the problem (11)-(3), they are often related to the approximation by the vanishing viscosity method with, say, a viscosity of (small) order $\varepsilon$. In [IV03, DIV03] the tools are developed (the notion of a kinetic solution for the initial boundary value problem) and the proof is given of an error estimate of order $\varepsilon^{1 / 3}$.

The article is structured as follows. In Section 2 we give and recall some properties of the entropy solution to the problem (1)-(3). In Section 3 the finite volume schemes under consideration are defined; some of their properties are explained in Section 4 while in Section 5 we prove the error estimates which are the center of our study. Finally, in Section [6] we give numerical experiments to illustrate our analysis. We complete the presentation with the proof of a BV estimate on the entropy solution on convex polygonal bounded domains in Appendix A.

\section{Properties And Regularity of the EXACT SOlUtion}

Problem (11)-(3) for general flux F, and in the context of entropy solutions, has first been analyzed by C. Bardos, A.-Y. LeRoux and J.-C. Nédélec [BLN79] in the $B V$ framework. The notion of entropy solution given by the three authors has been extended, in the $L^{\infty}$ setting, by F. Otto Ott96, MNRR96. We present and use this last definition by using the following semi-Kruzhkov entropy-entropy flux pairs Ser96, Car99.

Notation 2.1 (Semi-Kruzhkov entropy-entropy flux pairs). Let $a \top b$ (resp. $a \perp b$ ) denote the maximum (resp. the minimum) of $a$ and $b$, set $s^{+}=s \top 0, s^{-}=(-s)^{+}$ and denote by $\operatorname{sgn}_{ \pm}(s)$ the derivative of the function $s^{+}\left(\right.$resp. $\left.s^{-}\right)$with the value 0 at $s=0$. We denote by $\Phi^{ \pm}(s, \kappa)$ the entropy flux associated to the entropy $(s-\kappa)^{ \pm}$; that is to say

$$
\Phi^{ \pm}(x, t, s, \kappa)=\operatorname{sgn}_{ \pm}(s-\kappa)(\mathbf{F}(x, t, s)-\mathbf{F}(x, t, \kappa)) .
$$

We will often drop the dependence of $\Phi^{ \pm}$over the variables $x$ and $t$ and shorten the notation to $\Phi^{ \pm}(s, \kappa)$.

Notation 2.2. We denote by $C_{m}$ and $C_{M} \in \mathbb{R}$ some lower and upper bounds for the data

$$
C_{m} \leq c_{0}, \bar{c} \leq C_{M} \quad \text { a.e., }
$$

set $\mathcal{C}=\max \left(\left|C_{m}\right|,\left|C_{M}\right|\right)$ and let $\mathcal{L}$ be a fixed real satisfying

$$
\mathcal{L} \geq \max \left\{\left|\mathbf{F}_{c}(x, t, c)\right| ;(x, t, c) \in \Omega \times(0, T) \times\left[C_{m}, C_{M}\right]\right\} .
$$

Definition 2.3 (Entropy solution). A function $c \in L^{\infty}(\Omega \times(0, T))$ is called an entropy weak solution of (1)-(3) if it satisfies the following entropy inequalities: for all $\kappa \in\left[C_{m}, C_{M}\right]$, for all $\varphi \in C_{c}^{\infty}\left(\mathbb{R}^{d} \times \mathbb{R}^{+}\right)$with $\varphi \geq 0$,

$$
\begin{aligned}
\int_{\Omega \times(0, T)}(c-\kappa)^{ \pm} \partial_{t} \varphi+\Phi^{ \pm}(c, \kappa) & \cdot \nabla \varphi+\int_{\Omega}\left(c_{0}-\kappa\right)^{ \pm} \varphi(\cdot, 0) \\
& +\mathcal{L} \int_{\partial \Omega \times(0, T)}(\bar{c}-\kappa)^{ \pm} \varphi \geq 0 .
\end{aligned}
$$

The space $L^{\infty}$ is preserved by equation (1), as well as the space $B V$, and we have the following theorem. 
Theorem 2.4 (Existence, uniqueness, and regularity). Let $c_{0} \in L^{\infty}(\Omega), \bar{c} \in$ $L^{\infty}(\partial \Omega \times(0, T))$. Suppose that $\mathbf{F} \in \mathcal{C}^{1}(\Omega \times(0, T) \times \mathbb{R})$ and $\operatorname{div}_{x} \mathbf{F}(x, t, c)=0$ for all $(x, t, c) \in \Omega \times(0, T) \times \mathbb{R}$. Then there exists a unique entropy weak solution $c \in L^{\infty}(\Omega \times(0, T))$ of the problem (11) -(3) which is bounded by the data

$$
\|c(t)\|_{L^{\infty}(\Omega \times(0, T))} \leq \max \left\{\left\|c_{0}\right\|_{L^{\infty}(\Omega)},\|\bar{c}\|_{L^{\infty}(\partial \Omega \times(0, T))}\right\} .
$$

If, furthermore, $c_{0} \in B V(\Omega)$ and $\bar{c} \in B V(\partial \Omega \times(0, T))$, then $c \in B V(\Omega \times(0, T))$ and there exists a constant $C_{B V}>0$ which depends on the data and on $\Omega$ only such that

$$
\|c\|_{B V(\Omega \times(0, T))} \leq C_{B V}
$$

Proof. We refer to BLN79, Ott96, MNRR96, Vov02, for the results of existence and uniqueness of the entropy solution. In BLN79] a $B V$ estimate is given on the entropy solution, which requires $\Omega$ to be $\mathcal{C}^{2}$. The $B V$ estimate in the case where $\Omega$ is a polygonal bounded domain is a new result and we give the rather involved and technical proof in Appendix A.

Remark 2.5 (BLN). Under the hypotheses of Theorem 2.4 let $c$ be the entropy solution of the problem (11)-(3). Suppose that $c \in B V(\Omega \times(0, T))$ and denote by $\gamma c$ the trace of the function $c$ on $\partial \Omega \times(0, T)$. Then

(1) $c$ satisfies the following entropy inequalities for all $\kappa \in\left[C_{m}, C_{M}\right]$

$$
\begin{array}{r}
\int_{\Omega \times \mathbb{R}^{+}}(c-\kappa)^{ \pm} \partial_{t} \varphi+\Phi^{ \pm}(c, \kappa) \nabla \varphi+\int_{\Omega}\left(c_{0}-\kappa\right)^{ \pm} \varphi(\cdot, 0) \\
-\int_{\partial \Omega \times(0, T)} \Phi^{ \pm}(\gamma c, \kappa) \cdot \mathbf{n} \varphi \geq 0
\end{array}
$$

for all $\varphi \in C_{c}^{\infty}\left(\mathbb{R}^{d} \times \mathbb{R}^{+}\right)$with $\varphi \geq 0$.

(2) Moreover, the so-called BLN condition BLN79 is satisfied by $c$ on the boundary of the domain: for a.e. $(x, t) \in \partial \Omega \times(0, T)$, for all $\kappa$ in the interval with extremities $\gamma c(x, t)$ and $\bar{c}(x, t)$

$$
\Phi^{ \pm}(\gamma c, \kappa) \cdot \mathbf{n} \geq 0 .
$$

(3) The inequality (8) together with (9) implies (6). Indeed, if $(x, t) \in \partial \Omega \times$ $(0, T)$ and if $\kappa \leq \bar{c}(x, t)$, then (9) gives

$$
-\Phi^{-}(\gamma c(x, t), \kappa) \cdot \mathbf{n} \leq 0=\mathcal{L}(\bar{c}(x, t)-\kappa)^{-},
$$

while, if $\kappa>\bar{c}(x, t)$, then (9) gives $\Phi^{-}(\gamma c, \bar{c}) \cdot \mathbf{n} \geq 0$ and

${ }^{-} \Phi^{-}(\gamma c(x, t), \kappa) \cdot \mathbf{n} \leq\left(\Phi^{-}(\gamma c, \bar{c})-\Phi^{-}(\gamma c, \kappa)\right) \cdot \mathbf{n} \leq \mathcal{L}(\bar{c}(x, t)-\kappa)^{-}$.

(4) In fact, it is possible to prove (see [MNRR96, Vov02]) that for every function $w$ which is measurable and bounded a.e. on $\partial \Omega \times(0, T)$, one has

$$
-\Phi^{ \pm}(\gamma c(x, t), w(x, t)) \cdot \mathbf{n} \leq \mathcal{L}(\bar{c}(x, t)-w(x, t))^{ \pm}
$$

for a.e. $(x, t) \in \partial \Omega \times(0, T)$. 


\section{Notations, assumption And the Definition of the scheme}

In this section we will fix the notations and assumptions and define the finite volume scheme for solving (11)-(3).

Assumption 3.1. The data of problem (11)-(3) are supposed to satisfy the following conditions:

$$
\begin{array}{lll}
c_{0} \in L^{\infty} \cap B V\left(\mathbb{R}^{d}\right), & & \bar{c} \in L^{\infty} \cap B V(\partial \Omega \times(0, T)), \\
\mathbf{F} \in \mathcal{C}^{1}(\Omega \times(0, T) \times \mathbb{R}), & & \operatorname{div}_{x} \mathbf{F}(x, t, c)=0 \forall(x, t, c) \in \Omega \times(0, T) \times \mathbb{R} .
\end{array}
$$

The initial and boundary data are supposed to belong to the space $L^{\infty} \cap B V$. This makes sense if one has in mind practical applications in which these data are physical or biological quantities. The regularity and divergence-free hypotheses on the flux $F$ are also in coherence with the possible physical or biological underlying model for equation (11). The divergence free condition in (11) may be removed, and source terms may be considered in equation (11) as well.

Let us now give the description of the meshes and schemes used to solve (11)-(3). Let $J:=\left\{t_{0}, \ldots, t_{N}\right\}$ be a partition of $[0, T]$ and $\Delta t^{n}:=t^{n+1}-t^{n}$ be the step size of $J$. For each $n \in\{0, \ldots, N\}$, let $\mathcal{T}^{n}=\left\{T_{j} \mid j \in I_{\text {int }}^{n}\right\}$ be a regular triangulation of $\Omega$. The joint edge of $T_{j}$ and $T_{l}$ will be denoted by $S_{j l}$.

The set of internal edges $\mathcal{S}_{\text {int }}^{n}$ and the oriented set of internal edges $\mathcal{E}_{\text {int }}^{n}$ are assimilated to the sets of the corresponding indexes and are defined respectively as

$$
\begin{gathered}
\left.\mathcal{S}_{\text {int }}^{n}:=\left\{(j, l) \in I_{\text {int }}^{n} \times I_{\text {int }}^{n}\right\} \mid S_{j l} \text { is interior edge of } \mathcal{T}^{n}\right\}, \\
\mathcal{E}_{\text {int }}^{n}:=\left\{(j, l) \in \mathcal{S}_{\text {int }}^{n} \mid j>l\right\} .
\end{gathered}
$$

As mentioned in the Introduction, we use the concept of ghost cells to compute the flux at the boundary. Therefore, we introduce the notations related to the use of this method. Let the index set $I_{\text {ext }}^{n}$ be such that $I_{\text {ext }}^{n} \cap I_{\text {int }}^{n}=\emptyset$ and such that for each edge $S \subset \partial \Omega$ there exists a unique pair of indices $(j, l) \in I_{\text {int }}^{n} \times I_{\text {ext }}^{n}$ with $\partial T_{j} \cap S=S$. In this situation we denote $S_{j l}:=S$. Accordingly, the set of edges located on the boundary of $\Omega$ is denoted by

$$
\mathcal{S}_{\text {ext }}^{n}:=\left\{(j, l) \in I_{\text {int }}^{n} \times I_{\text {ext }}^{n} \mid S_{j l} \text { is an exterior edge of } \mathcal{T}^{n}\right\} .
$$

We also denote by $h_{\min }^{n}:=\min _{j \in I^{n}} \operatorname{diam}\left(T_{j}\right)$ the size of the mesh at time $t^{n}$. The mesh $\mathcal{T}^{n}$ satisfies the following structural hypothesis.

Assumption 3.2. There exists a real $\alpha>0$ such that for all $h_{j}:=\operatorname{diam}\left(T_{j}\right), j \in I$,

$$
\alpha h_{j}^{d} \leq\left|T_{j}\right|, \quad \alpha\left|\partial T_{j}\right| \leq h_{j}^{d-1} .
$$

In order to design the finite volume approximation, we first define the class of monotone numerical fluxes in use.

Definition 3.3 (Numerical fluxes). The numerical fluxes $g_{j l}^{n}$ are functions in $\mathcal{C}\left(\mathbb{R}^{2}, \mathbb{R}\right)$, for any $(j, l) \in \mathcal{S}^{n}$ and $t_{n} \in \mathbb{R}^{+}$, satisfying the following conditions 
(respectively: monotony, convervativity, regularity, consistency).

$$
\begin{aligned}
& \forall v \in\left[C_{m}, C_{M}\right], \quad g_{j l}^{n}(v, \cdot) \text { is monotone nonincreasing on }\left[C_{m}, C_{M}\right], \\
& \forall w \in\left[C_{m}, C_{M}\right], \quad g_{j l}^{n}(\cdot, w) \text { is monotone nondecreasing on }\left[C_{m}, C_{M}\right], \\
& \forall v, w \in\left[C_{m}, C_{M}\right], \forall(j, l) \in \mathcal{S}_{\text {int }}^{n}, \quad g_{j l}^{n}(v, w)=-g_{l j}^{n}(w, v),
\end{aligned}
$$

$$
\forall w, v, w^{\prime}, v^{\prime} \in\left[C_{m}, C_{M}\right], \quad\left|g_{j l}^{n}(v, w)-g_{j l}^{n}\left(v^{\prime}, w^{\prime}\right)\right| \leq \mathcal{L}\left|S_{j l}\right|\left(\left|w-w^{\prime}\right|+\left|v-v^{\prime}\right|\right),
$$

and

$$
g_{j l}^{n}(w, w)=\frac{1}{\Delta t} \int_{t^{n}}^{t^{n+1}} \int_{S_{j l}} \mathbf{F}(x, t, w) \cdot n_{j l} \mathrm{dx} \mathrm{dt},
$$

where $n_{j l}$ denotes the outer unit normal to $S_{j l}$ with respect to $T_{j}$.

Definition 3.4 (Finite volume scheme). Set

$c_{j}^{0}:=\frac{1}{\left|T_{j}\right|} \int_{T_{j}} c_{0}, j \in I_{\text {int }}^{0}, \quad c_{l}^{n}:=\frac{1}{\Delta t} \frac{1}{\left|S_{j l}\right|} \int_{t^{n}}^{t^{n+1}} \int_{S_{j, l}} \bar{c}(x, t) \mathrm{dx} \mathrm{dt},(j, l) \in \mathcal{S}_{\text {ext }}^{n}$.

The discrete evolution of the approximate value $c_{j}$ of $c$ in the cell $T_{j}$ is governed by the equation

$$
c_{j}^{n+1}:=c_{j}^{n}-\frac{\Delta t}{\left|T_{j}\right|} \sum_{l \in N(j)} g_{j l}^{n}\left(c_{j}^{n}, c_{l}^{n}\right), \quad j \in I_{\mathrm{int}}^{n},
$$

for all $n \in\{0, \ldots, N\}$, where $N(j)$ denotes the index set of the neighboring cells of $T_{j}$, including the ghost indices across the boundaries of the domain $\Omega$.

Given the discrete values $c_{j}^{n}$, we denote by $c_{h}$ the approximate solution $c_{h}: \Omega \times(0, T) \rightarrow \mathbb{R}$ defined by

$$
c_{h}(x, t):=c_{j}^{n} \quad \text { if } \quad x \in T_{j}, \quad t^{n} \leq t<t^{n+1} .
$$

The stability of the explicit scheme (17) is ensured under the following CFL condition.

Assumption 3.5 (CFL condition). We assume the following CFL condition for a given $\xi \in(0,1)$ :

$$
\Delta t^{n} \leq \frac{(1-\xi) \alpha^{2} h_{\min }^{n}}{\mathcal{L}}
$$

\section{Properties of the Discrete solution}

As the entropy solution of problem (11)-(3), the discrete solution is $L^{\infty}$ stable. On the contrary, the validity of $B V$ estimates on $c_{h}$ is still an open question (in the case where unstructured meshes are considered): only "weak $B V$ estimates" are known. These two aspects of the behavior of the discrete solution are detailed in the following two lemmas (see Vov02 for a proof).

Lemma 4.1 ( $L^{\infty}$ stability). Let $c_{h}$ be the discrete solution (17), and let the Assumptions 3.1, 3.2, and 3.5 be fulfilled. Then the function $c_{h}$ satisfies the $L^{\infty}$ estimates,

and

$$
\left\|c_{h}\right\|_{L^{\infty}(\Omega \times[0, T])} \leq \max \left\{\left\|c_{0}\right\|_{L^{\infty}(\Omega)},\|\bar{c}\|_{L^{\infty}(\partial \Omega \times(0, T))}\right\}
$$

$$
C_{m} \leq c_{j}^{n} \leq C_{M}, \quad \text { for all }\left(T_{j}, t^{n}\right) \in \mathcal{T}^{n} \times J \text {. }
$$


Lemma 4.2 (Weak BV estimate). Let $c_{h}$ be the discrete solution (17) and let the Assumptions 3.1, 3.2, and 3.5 be fulfilled. Then there exists $C \geq 0$ only depending on $\Omega, c_{0}, \bar{c}, \mathcal{L}, T, \alpha$ and $\xi$ such that

$$
\begin{aligned}
\sum_{t^{n} \in J(j, l) \in \mathcal{E}_{\text {int }}^{n}} \Delta t^{n}( & \max _{c_{l}^{n} \leq a \leq b \leq c_{j}^{n}}\left(g_{j l}^{n}(b, a)-g_{j l}^{n}(b, b)\right) \\
& +\max _{c_{l}^{n} \leq a \leq b \leq c_{j}^{n}}\left(g_{j l}^{n}(b, a)-g_{j l}^{n}(a, a)\right) \leq \frac{C}{\sqrt{h}},
\end{aligned}
$$

and

$$
\sum_{t^{n} \in J} \sum_{j \in I^{n}}\left|T_{j}\right|\left|c_{j}^{n+1}-c_{j}^{n}\right| \leq \frac{C}{\sqrt{h}} .
$$

Entropy inequality satisfied by the approximate solution. In Section 2 we recalled that problem (1) has a unique weak solution conforming to the entropy inequality (6). In this subsection we will show that the approximate solution $c_{h}$ fulfills an analog inequality, including a small error term. To compare discrete to continuous equations, let us introduce the following forms $E^{+}$and $E_{h}^{+}$.

Definition 4.3. For the discrete function $c_{h}$ being defined by (3.4) and $\kappa \in \mathbb{R}$, we set

$$
\begin{aligned}
E_{h}^{+}\left(c_{h}, \kappa, \varphi\right):= & -\sum_{t^{n} \in J j \in I^{n}} \frac{\left(c_{j}^{n+1}-\kappa\right)^{+}-\left(c_{j}^{n}-\kappa\right)^{+}}{\Delta t^{n}} \int_{t^{n}}^{t^{n+1}} \int_{T_{j}} \varphi(x, t) \mathrm{dxdt} \\
& -\sum_{t^{n} \in J j \in I^{n}} \sum_{j} \frac{1}{\left|T_{j}\right|} \int_{t^{n}}^{t^{n+1}} \int_{T_{j}} \varphi(x, t) \sum_{l \in N(j)} g_{j l}^{n}\left(c_{j}^{n} \top \kappa, c_{l}^{n} \top \kappa\right)-g_{j l}^{n}(\kappa, \kappa) \mathrm{dxdt}, \\
E^{+}\left(c_{h}, \kappa, \varphi\right):= & \int_{\Omega \times \mathbb{R}^{+}}\left(c_{h}(x, t)-\kappa\right)^{+} \partial_{t} \varphi(x, t) \mathrm{dxdt} \\
& +\int_{\Omega \times \mathbb{R}^{+}} \Phi^{+}\left(c_{h}(x, t), \kappa\right) \cdot \nabla \varphi(x, t) \mathrm{dxdt} \\
& +\int_{\Omega}\left(c_{0}(x)-\kappa\right)^{+} \varphi(x, 0) \mathrm{dx}+\mathcal{L} \int_{\partial \Omega \times(0, T)}(\bar{c}(x, t)-\kappa)^{+} \varphi \mathrm{d} \gamma(x) \mathrm{dt},
\end{aligned}
$$

for any $\varphi \in \mathcal{C}^{\infty}(\bar{\Omega} \times[0, T))$.

The discrete (and local) entropy inequality given in Lemma 4.4 is the main account for the approximate continuous entropy inequality detailed in Lemma 4.5.

Lemma 4.4 (Discrete entropy inequality). Let $c_{h}$ be the discrete solution defined in 3.4, and let Assumptions 3.1, 3.2, and 3.5 be fulfilled. Then we have

$$
E_{h}^{+}\left(c_{h}, \kappa, \varphi\right) \geq 0 .
$$

Proof. The discrete entropy inequality (21) follows from the monotonicity properties of the numerical fluxes; see, e.g., Vov02.

Lemma 4.5 (Continuous entropy estimate). Let $c_{h}$ be the discrete solution defined in Definition 3.4, and let the Assumptions 3.1, 3.2, and 3.5 be fulfilled. Then we 
have

$$
\begin{aligned}
E^{+}\left(c_{h}, \kappa, \varphi\right) \geq & -\int\left|c c_{h}(x, 0)-c_{0}(x)\right| \varphi(x, 0) d x \\
& -\sum_{t^{n} \in J j \in I^{n}}\left|c_{j}^{n+1}-c_{j}^{n}\right| \int_{T_{j}} \int_{t^{n}}^{t^{n+1}}\left|\varphi_{t}(x, t)\right| d x d t \\
& -\sum_{t^{n} \in J(j, l) \in \mathcal{E}_{\text {int }}^{n}} 2 \max _{c_{l}^{n} \leq a \leq b \leq c_{j}^{n}}\left(g_{j l}^{n}(b, a)-g_{j l}^{n}(b, b)\right)\left\langle\mu_{j l}^{n},|\nabla \varphi|+\left|\varphi_{t}\right|\right\rangle \\
& -\sum_{t^{n} \in J(j, l) \in \mathcal{E}_{\text {int }}^{n}} 2 \max _{c_{l}^{n} \leq a \leq b \leq c_{j}^{n}}\left(g_{j l}^{n}(b, a)-g_{j l}^{n}(a, a)\right)\left\langle\mu_{l j}^{n},|\nabla \varphi|+\left|\varphi_{t}\right|\right\rangle \\
& -\sum_{t^{n} \in J(j, l) \in \mathcal{E}_{\text {int }}^{n}} \sum_{\left(\left|c_{j}^{n}\right|+\left|c_{l}^{n}\right|\right)\left\langle\nu_{j l}^{n},|\nabla \varphi|+\left|\varphi_{t}\right|\right\rangle} \\
& -\mathcal{L} \int_{\partial \Omega \times(0, T)}\left(\bar{c}_{h}-\bar{c}\right)^{+} \varphi(x, t) d \gamma(x) d t \\
& -\sum_{t^{n} \in J(j, l) \in \mathcal{S}_{\text {ext }}^{n}} \sum \mathcal{L}\left(c_{l}+c_{j}-2 C_{m}\right)\left\langle\bar{\nu}_{l j}^{n},|\nabla \varphi|\right\rangle,
\end{aligned}
$$

where the Radon measures $\mu_{j l}^{n}, \nu_{j l}^{n}, \bar{\nu}_{j l}^{n}$ are defined as

$$
\begin{array}{rl}
\left\langle\mu_{j l}^{n}, g\right\rangle:=\frac{h_{j}+\Delta t^{n}}{\Delta t^{n}\left|T_{j}\right|\left|S_{j l}\right|} \int_{t^{n}}^{t^{n+1}} \int_{T_{j}} \int_{t^{n}}^{t^{n+1}} \int_{S_{j l}} \int_{0}^{1} & g(\gamma+\vartheta(x-\gamma), s+\vartheta(t-s)) d \vartheta d \gamma d s d x d t, \\
\left\langle\nu_{j l}^{n}, g\right\rangle:=\frac{\left(h_{j}+\Delta t^{n}\right)^{2}}{\Delta t^{n}\left|S_{j l}\right|} \int_{t^{n}}^{t^{n+1}} \int_{S_{j l}} \int_{t^{n}}^{t^{n+1}} \int_{S_{j l}} \int_{0}^{1} & g(\xi+\vartheta(\gamma-\xi), \tau+\vartheta(s-\tau)) d \vartheta d \gamma d t d \xi d \tau, \\
\left\langle\bar{\nu}_{j l}^{n}, g\right\rangle:=\frac{h_{j}}{\left|T_{j}\right|} \int_{t^{n}}^{t^{n+1}} \int_{T_{j}} \int_{S_{j l}} \int_{0}^{1} g(\gamma+\vartheta(x-\gamma), t) d \vartheta d \gamma d x d t .
\end{array}
$$

Here, we have introduced the discrete boundary datum $\bar{c}_{h}$ defined by $\left.\bar{c}_{h}\right|_{S_{j l} \times\left[t^{n}, t^{n+1}\right)}$ $:=c_{l}^{n}$ for $(j, l) \in \mathcal{S}_{\text {ext }}^{n}$.

Proof. From Lemma 4.4 follows

$$
E^{+}\left(c_{h}, \kappa, \varphi\right) \geq E^{+}\left(c_{h}, \kappa, \varphi\right)-E_{h}^{+}\left(c_{h}, \kappa, \varphi\right)
$$

Hence, we aim at deriving a lower estimate for $E^{+}\left(c_{h}, \kappa, \varphi\right)-E_{h}^{+}\left(c_{h}, \kappa, \varphi\right)$. In order to do so, we split the addend on the right-hand side into two terms, corresponding to the time derivative and the convection part. This yields

$$
E^{+}\left(c_{h}, \kappa, \varphi\right)-E_{h}^{+}\left(c_{h}, \kappa, \varphi\right)=\left(T_{10}-T_{1}\right)+\left(T_{20}-T_{2}\right),
$$


where the terms $T_{1}, T_{10}, T_{2}, T_{20}$ are defined by

$$
\begin{aligned}
T_{1}= & -\sum_{t^{n} \in J} \sum_{j \in I^{n}} \frac{\left(c_{j}^{n+1}-\kappa\right)^{+}-\left(c_{j}^{n}-\kappa\right)^{+}}{\Delta t^{n}} \int_{t^{n}}^{t^{n+1}} \int_{T_{j}} \varphi(x, t) \mathrm{dxdt}, \\
T_{10}= & \int_{\Omega \times \mathbb{R}^{+}}\left(c_{h}(x, t)-\kappa\right)^{+} \partial_{t} \varphi(x, t),+\int_{\Omega}\left(c_{0}(x)-\kappa\right)^{+} \varphi(x, 0) \mathrm{dx}, \\
T_{2}= & -\sum_{t^{n} \in J j \in I^{n}} \sum_{\left|T_{j}\right|} \int_{t^{n}}^{t^{n+1}} \int_{T_{j}} \varphi(x, t) \sum_{l \in N(j)}\left(g_{j l}^{n}\left(c_{j}^{n} \top \kappa, c_{l}^{n} \top \kappa\right)-g_{j l}^{n}(\kappa, \kappa)\right), \\
T_{20}= & \int_{\Omega \times \mathbb{R}^{+}}\left(\mathbf{F}\left(x, t, c_{h}(x, t) \top \kappa\right)-\mathbf{F}(x, t, \kappa)\right) \nabla \varphi(x, t) \mathrm{dx} \mathrm{dt} \\
& -\int_{\partial \Omega \times(0, T)} \widetilde{\gamma \Phi_{h}^{+}}\left(c_{h}, \bar{c}, \kappa\right) \varphi(x, t) \mathrm{d} \gamma(x) \mathrm{dt} .
\end{aligned}
$$

From (11) and (16) it follows that

$$
\begin{aligned}
T_{2}= & -\sum_{t^{n} \in J} \sum_{j \in I^{n}} \frac{1}{\left|T_{j}\right|} \int_{t^{n}}^{t^{n+1}} \int_{T_{j}} \varphi(x, t) \\
& \times \sum_{l \in N(j)}\left\{\left(g_{j l}^{n}\left(c_{j}^{n} \top \kappa, c_{l}^{n} \top \kappa\right)-g_{j l}^{n}(\kappa, \kappa)\right),-\left(g_{j l}^{n}\left(c_{j}^{n} \top \kappa, c_{j}^{n} \top \kappa\right)-g_{j l}^{n}(\kappa, \kappa)\right)\right\} .
\end{aligned}
$$

The discrete function $c_{h}$ is piecewise constant in space and time. Thus, we decompose $T_{20}$ into sums and integrate by parts locally:

$$
\begin{array}{r}
T_{20}=\sum_{t^{n} \in J} \sum_{j \in I^{n}} \int_{t^{n}}^{t^{n+1}} \int_{S_{j l}}\left(\mathbf{F}\left(x, t, c_{j} \top \kappa\right)-\mathbf{F}(x, t, \kappa)\right) \cdot n_{j l} \varphi(x, t) \mathrm{d} \gamma(x) \mathrm{dt} \\
-\int_{\partial \Omega \times(0, T)} \widetilde{\gamma \Phi_{h}^{+}}\left(c_{h}, \bar{c}, \kappa\right) \varphi(x, t) \mathrm{d} \gamma(x) \mathrm{dt} .
\end{array}
$$

Next we have the summation in $T_{20}$, and then $T_{2}$ is rearranged in accordance to the following lemma.

Lemma 4.6. Let $A$ be a function $\prod_{n \in \mathbb{N}}\{n\} \times \mathcal{S}^{n} \rightarrow \mathbb{R}$. Then

$$
\sum_{t^{n} \in J} \sum_{j \in I^{n}} \sum_{l \in N(j)} A_{j l}^{n}=\sum_{t^{n} \in J} \sum_{(j, l) \in \mathcal{E}_{\mathrm{int}}^{n}}\left(A_{j l}^{n}+A_{l j}^{n}\right)+\sum_{t^{n} \in J(j, l) \in \mathcal{S}_{\mathrm{ext}}^{n}} A_{j l}^{n}
$$

This reordering of the summations in $T_{2}$ and $T_{20}$ on the edges leads to the decomposition $T_{2}=T_{2}^{\text {int }}+T_{2}^{\text {ext }}$ and $T_{20}=T_{20}^{\text {int }}+T_{20}^{\text {ext }}$. Then the method used to estimate the term $\left|T_{20}^{\text {int }}-T_{2}^{\text {int }}\right|$, as well as the term $\left|T_{10}-T_{1}\right|$, is, step by step, the method used in the proof of Theorem 4 in [CH99a. or Theorem 4.1 in EGGH98. 
We refer to these articles for an integral proof of the following results:

$$
\begin{aligned}
\left|T_{10}-T_{1}\right| \leq & \sum_{t^{n} \in J j \in I^{n}} \sum_{j}\left|c_{j}^{n+1}-c_{j}^{n}\right| \int_{T_{j}} \int_{t^{n}}^{t^{n+1}}\left|\varphi_{t}(x, t)\right| \mathrm{dxdt} \\
& +\int_{\Omega}\left|c_{h}(x, 0)-c_{0}(x)\right| \varphi(x, 0) \mathrm{dx}, \\
\left|T_{20}^{\text {int }}-T_{2}^{\mathrm{int}}\right| \leq & \sum_{t^{n} \in J(j, l) \in \mathcal{E}_{\text {int }}^{n}} \sum_{\mathcal{L}}\left(\left|c_{j}^{n}\right|+\left|c_{l}^{n}\right|\right)\left\langle\nu_{j l}^{n},|\nabla \varphi|+\left|\varphi_{t}\right|\right\rangle \\
& +\sum_{t^{n} \in J(j, l) \in \mathcal{E}_{\text {int }}^{n}} \sum_{c_{l}^{n} \leq a \leq b \leq c_{j}^{n}}\left(g_{j l}^{n}(b, a)-g_{j l}^{n}(b, b)\right)\left\langle\mu_{j l}^{n},|\nabla \varphi|+\left|\varphi_{t}\right|\right\rangle \\
& +\sum_{t^{n} \in J(j, l) \in \mathcal{E}_{\text {int }}^{n}} \sum_{c_{l}^{n} \leq a \leq b \leq c_{j}^{n}}\left(g_{j l}^{n}(b, a)-g_{j l}^{n}(a, a)\right)\left\langle\mu_{l j}^{n},|\nabla \varphi|+\left|\varphi_{t}\right|\right\rangle,
\end{aligned}
$$

where $\mu_{j l}^{n}$ and $\nu_{j l}^{n}$ are defined in the statement of the lemma.

A lower estimate on the boundary terms $T_{20}^{\text {ext }}-T_{2}^{\text {ext }}$ remains to be proved. We have

$$
\begin{aligned}
T_{2}^{\mathrm{ext}}= & -\sum_{t^{n} \in J(j, l) \in \mathcal{S}_{\mathrm{ext}}^{n}} \frac{1}{\left|T_{j}\right|} \int_{t^{n}}^{t^{n+1}} \int_{T_{j}} \varphi(x, t)\left\{\left(g_{j l}^{n}\left(c_{j}^{n} \top \kappa, c_{l}^{n} \top \kappa\right)-g_{j l}^{n}(\kappa, \kappa)\right),\right. \\
& \left.-\left(g_{j l}^{n}\left(c_{j}^{n} \top \kappa, c_{j}^{n} \top \kappa\right)-g_{j l}^{n}(\kappa, \kappa)\right)\right\}, \\
T_{20}^{\mathrm{ext}}= & \sum_{t^{n} \in J(j, l) \in \mathcal{S}_{\mathrm{ext}}^{n}} \int_{t^{n}} \int_{S_{j l}}\left(\mathbf{F}\left(x, t, c_{j} \top \kappa\right)-\mathbf{F}(x, t, \kappa)\right) \cdot n_{j l} \varphi(x, t) \mathrm{d} \gamma(x) \mathrm{dt} \\
& +\mathcal{L} \int_{\partial \Omega \times(0, T)}(\bar{c}-\kappa)^{+} \varphi(x, t) \mathrm{d} \gamma(x) \mathrm{dt} .
\end{aligned}
$$

First, observe that, by (16), we have

$$
\begin{aligned}
-\sum_{t^{n} \in J(j, l) \in \mathcal{S}_{\text {ext }}^{n}} & \frac{1}{\left|T_{j}\right|} \int_{t^{n}}^{t^{n+1}} \int_{T_{j}} \varphi(x, t)\left(g_{j l}^{n}\left(c_{j}^{n} \top \kappa, c_{j}^{n} \top \kappa\right)-g_{j l}^{n}(\kappa, \kappa)\right) \mathrm{dx} \mathrm{dt}, \\
= & -\sum_{t^{n} \in J(j, l) \in \mathcal{S}_{\text {ext }}^{n}} \int_{t^{n}} \int_{S_{j l}}\left(\mathbf{F}\left(x, t, c_{j} \top \kappa\right)-\mathbf{F}(x, t, \kappa)\right) \cdot n_{j l} \varphi_{j}^{n} \mathrm{~d} \gamma(x) \mathrm{d} t,
\end{aligned}
$$

where $\varphi_{j}^{n}:=\frac{1}{\left|T_{j}\right|} \int_{t^{n}}^{t^{n+1}} \int_{T_{j}} \varphi(x, t) \mathrm{dxdt}$, and hence

$$
\begin{aligned}
T_{20}^{\text {ext }}- & T_{2}^{\text {ext }}=\sum_{t^{n} \in J(j, l) \in \mathcal{S}_{\text {ext }}^{n}} \frac{1}{\left|T_{j}\right|} \int_{t^{n}}^{t^{n+1}} \int_{T_{j}} \varphi(x, t)\left(g_{j l}^{n}\left(c_{j}^{n} \top \kappa, c_{l}^{n} \top \kappa\right)-g_{j l}^{n}(\kappa, \kappa)\right) \\
& \sum_{t^{n} \in J(j, l) \in \mathcal{S}_{\text {ext }}^{n}} \int_{t^{n}} \int_{S_{j l}}\left(\mathbf{F}\left(x, t, c_{j} \top \kappa\right)-\mathbf{F}(x, t, \kappa)\right) \cdot n_{j l}\left(\varphi(x, t)-\varphi_{j}^{n}\right) \mathrm{d} \gamma(x) \mathrm{dt} \\
& +\mathcal{L} \int_{\partial \Omega \times(0, T)}(\bar{c}-\kappa)^{+} \varphi(x, t) \mathrm{d} \gamma(x) \mathrm{dt} \\
=: & U_{1}^{\text {ext }}+U_{2}^{\text {ext }}+U_{3}^{\text {ext }}+U_{4}^{\text {ext }},
\end{aligned}
$$


where

$$
\begin{aligned}
U_{1}^{\text {ext }}:= & \sum_{t^{n} \in J(j, l) \in \mathcal{S}_{\text {ext }}^{n}} \frac{1}{\left|T_{j}\right|} \int_{t^{n}}^{t^{n+1}} \int_{T_{j}} \varphi(x, t)\left(g_{j l}^{n}\left(c_{j}^{n} \top \kappa, c_{l}^{n} \top \kappa\right)-g_{j l}^{n}(\kappa, \kappa)\right) \\
& -\sum_{t^{n} \in J(j, l) \in \mathcal{S}_{\text {ext }}^{n}} \frac{1}{\left|S_{j l}\right|} \int_{t^{n}}^{t^{n+1}} \int_{S_{j l}} \varphi(x, t)\left(g_{j l}^{n}\left(c_{j}^{n} \top \kappa, c_{l}^{n} \top \kappa\right)-g_{j l}^{n}(\kappa, \kappa)\right), \\
U_{2}^{\text {ext }}:= & \sum_{t^{n} \in J(j, l) \in \mathcal{S}_{\text {ext }}^{n}} \int_{t^{n}} \int_{S_{j l}}^{t^{n+1}} \\
& \times\left(\mathbf{F}\left(x, t, c_{j} \top \kappa\right)-\mathbf{F}(x, t, \kappa)\right) \cdot n_{j l}\left(\varphi(x, t)-\varphi_{j}^{n}\right) \mathrm{d} \gamma(x) \mathrm{dt}, \\
U_{3}^{\text {ext }}:= & \sum_{t^{n} \in J(j, l) \in \mathcal{S}_{\text {ext }}^{n}} \frac{1}{\left|S_{j l}\right|} \int_{t^{n}}^{t^{n+1}} \int_{S_{j l}} \varphi(x, t)\left(g_{j l}^{n}\left(c_{j}^{n} \top \kappa, c_{l}^{n} \top \kappa\right)-g_{j l}^{n}(\kappa, \kappa)\right) \\
& +\mathcal{L} \int_{\partial \Omega \times(0, T)}\left(\bar{c}_{h}-\kappa\right)^{+} \varphi(x, t) \mathrm{d} \gamma(x) \mathrm{dt}, \\
U_{4}^{\text {ext }:=} & \mathcal{L} \int_{\partial \Omega \times(0, T)}(\bar{c}-\kappa)^{+} \varphi(x, t) \mathrm{d} \gamma(x) \mathrm{dt} \\
& -\mathcal{L} \int_{\partial \Omega \times(0, T)}\left(\bar{c}_{h}-\kappa\right)^{+} \varphi(x, t) \mathrm{d} \gamma(x) \mathrm{dt} .
\end{aligned}
$$

Recall that the discrete boundary datum $\bar{c}_{h}$ is defined by $\left.\bar{c}_{h}\right|_{S_{j l} \times\left[t^{n}, t^{n+1}\right)}:=c_{l}^{n}$ for $(j, l) \in \mathcal{S}_{\text {ext }}^{n}$ which yields

$$
\int_{\partial \Omega \times(0, T)}\left(\bar{c}_{h}-\kappa\right)^{+} \varphi(x, t) \mathrm{d} \gamma(x) \mathrm{dt}=\sum_{t^{n} \in J(j, l) \in \mathcal{S}_{\mathrm{ext}}^{n}} \frac{1}{\left|S_{j l}\right|} \int_{t^{n}}^{t^{n+1}} \int_{S_{j l}} \varphi(x, t)\left(c_{l}^{n} \top \kappa-\kappa\right)
$$

and

$U_{3}^{\mathrm{ext}}=\sum_{t^{n} \in J(j, l) \in \mathcal{S}_{\mathrm{ext}}^{n}} \frac{1}{S_{j l} \mid} \int_{t^{n}}^{t^{n+1}} \int_{S_{j l}} \varphi(x, t)\left(g_{j l}^{n}\left(c_{j}^{n} \top \kappa, c_{l}^{n} \top \kappa\right)-g_{j l}^{n}(\kappa, \kappa)+\mathcal{L}\left(c_{l}^{n} \top \kappa-\kappa\right)\right)$.

Since $g_{j l}^{n}$ is nondecreasing with respect to its first variable, and by (15) we have

$$
g_{j l}^{n}\left(c_{j}^{n} \top \kappa, c_{l}^{n} \top \kappa\right)-g_{j l}^{n}(\kappa, \kappa) \geq g_{j l}^{n}\left(\kappa, c_{l}^{n} \top \kappa\right) \geq-\mathcal{L}\left(c_{l}^{n} \top \kappa-\kappa\right),
$$

we have $U_{3}^{\text {ext }} \geq 0$. Besides, using the 1-Lipschitz continuity of the function $c \mapsto$ $(c-\kappa)^{+}$and the $\mathcal{L}$-Lipschitz continuity of the numerical fluxes we derive the estimates

$$
\begin{aligned}
& U_{1}^{\text {ext }} \geq-\sum_{t^{n} \in J(j, l) \in \mathcal{S}_{\text {ext }}^{n}} \mathcal{L}\left(c_{l}-C_{m}\right)\left\langle\bar{\nu}_{l j}^{n},|\nabla \varphi|\right\rangle, \\
& U_{2}^{\text {ext }} \geq-\sum_{t^{n} \in J(j, l) \in \mathcal{S}_{\text {ext }}^{n}} \mathcal{L}\left(c_{j}-C_{m}\right)\left\langle\bar{\nu}_{l j}^{n},|\nabla \varphi|\right\rangle, \\
& U_{4}^{\text {ext }} \geq-\mathcal{L} \int_{\partial \Omega \times(0, T)}\left(\bar{c}_{h}-\bar{c}\right)^{+} \varphi(x, t) \mathrm{d} \gamma(x) \mathrm{dt} .
\end{aligned}
$$

This concludes the proof of Lemma 4.5 . 


\section{ERror estimates}

Proposition 5.1 (A posteriori estimate). Let $c_{h}$ be the discrete solution defined in Definition 3.4, and let $c$ be the entropy solution of (1)-(3). Furthermore, let the Assumptions 3.1, 3.2, and 3.5 be fulfilled. Then we have

$$
\left\|c_{h}-c\right\|_{L^{1}(\Omega \times(0, T))} \leq \eta\left(c_{h}\right),
$$

with

$\eta\left(c_{h}\right):=2 T\left(N_{f}+1\right) \min _{r, r_{d} \in \mathbb{R}^{+}}\left[\eta_{0}+\bar{\eta}+\left(\eta_{t}+\eta_{c}\right) K_{1} r+\eta_{c} K_{1}^{\prime}\left(r+r_{d}\right)+K_{0}\left(\frac{2}{r}+\frac{r}{r_{d}}\right)\right]$.

Here $\eta_{0}, \eta_{t}, \eta_{c}$, and $\bar{\eta}$ are defined by

$$
\begin{aligned}
\eta_{0}= & \int_{\Omega}\left|c_{h}(x, 0)-c_{0}(x)\right| d x, \\
\eta_{t}= & \sum_{t^{n} \in J j \in I^{n}} \sum_{j}\left|T_{j}\right| \Delta t^{n}\left|c_{j}^{n+1}-c_{j}^{n}\right|, \\
\eta_{c}= & \sum_{t^{n} \in J(j, l) \in \mathcal{E}_{\text {int }}^{n}}\left[2 ( h _ { j l } + \Delta t ^ { n } ) \Delta t ^ { n } \left(\max _{c_{l}^{n} \leq a \leq b \leq c_{j}^{n}}\left(g_{j l}^{n}(b, a)-g_{j l}^{n}(b, b)\right)\right.\right. \\
& \left.+\max _{c_{l}^{n} \leq a \leq b \leq c_{j}^{n}}\left(g_{j l}^{n}(b, a)-g_{j l}^{n}(a, a)\right)\right) \\
& \left.+\mathcal{L}\left(\left|c_{j}^{n}\right|+\left|c_{l}^{n}\right|\right)\left(h_{j l}+\Delta t^{n}\right)^{2} \Delta t^{n} h_{j l}\right] \\
& +\sum_{t^{n} \in J(j, l) \in \mathcal{S}_{\text {ext }}^{n}} \mathcal{L}\left(c_{j}+c_{l}-2 C_{m}\right) h_{j} \Delta t^{n} h_{j l}, \\
\bar{\eta}= & \int_{\partial \Omega \times(0, T)}\left|\bar{c}_{h}-\bar{c}\right| d \gamma(x) d t,
\end{aligned}
$$

and the constants $K_{1}, K_{1}^{\prime}$ are defined by (30). We have set

(23) $K_{0}:=2 d \mathcal{L C}\left(1+|| w_{0}^{\prime} \|_{L^{1}(\mathbb{R})}+\left|c_{0}\right|_{B V}+|\bar{c}|_{B V}+K_{1}^{\prime}+C_{B V}+C_{\Omega}\right)(1+T|\Omega|)$.

Proposition 5.2 (A priori estimate). Let $c_{h}$ be the discrete solution defined in Definition 3.4, and let $c$ be the entropy solution of (11)-(3). Furthermore, let the Assumptions 3.1, 3.2, and 3.5 be fulfilled. For uniform meshes of mesh size $h$ we then have the a priori error estimate

$$
\left\|c_{h}-c\right\|_{L^{1}(\Omega \times(0, T))} \leq \eta\left(c_{h}\right) \leq K h^{1 / 6} .
$$

Here $K$ denotes a generic constant independent of the mesh size.

The proof of the error estimates relies on the technique of the doubling of variables [Kru70, Kuz76]. The basic elements of this technique (approximation of the unit, choice of the test function and the so-called "dual forms") are the object of the following three definitions.

Definition 5.3 (Approximation of the unit). Let $w_{0} \in C_{0}^{\infty}(\mathbb{R}, \mathbb{R})$ be such that

$$
\operatorname{supp}\left(w_{0}\right) \subset[0,1], \quad w_{0} \geq 0, \quad \int_{\mathbb{R}} w_{0}(x) \mathrm{dx}=1 .
$$

For $r$ and $r_{d} \geq 1$, we define the approximation of the unit $w$ by $\forall(x, t)=\left(\bar{x}, x_{d}, t\right) \in$ $\mathbb{R}^{d} \times(0, T)$,

$$
w\left(\bar{x}, x_{d}, t\right)=r w_{0}\left(r x_{1}\right) \cdots w_{0}\left(r x_{d-1}\right) \times r_{d} w_{0}\left(r_{d} x_{d}\right) \times r w_{0}(r t) .
$$


Definition 5.4 (Choice of the test function). Let $\psi: \mathbb{R}^{d} \times \mathbb{R}^{+} \rightarrow \mathbb{R}^{+}$and $\lambda: \mathbb{R}^{d} \rightarrow$ $[0,1]$ denote some smooth nonnegative functions that will be specified later. We suppose $\operatorname{supp}(\psi) \subset \mathbb{R}^{d} \times[0, T)$. We set

$$
\varphi(x, t)=\varphi(x, t, y, s):=\psi(y, s) \lambda(y) w(x-y, t-s) .
$$

Remark 5.5. Notice that if $\alpha \in\left\{x_{1}, \ldots, x_{d-1}, t\right\}$, say $\alpha=t$ for example, then $\partial_{t} w(x, t)=r w_{0}\left(r x_{1}\right) \cdots w_{0}\left(r x_{d-1}\right) \times r_{d} w_{0}\left(r_{d} x_{d}\right) \times r^{2} w_{0}^{\prime}(r t)$ and, since $\int_{\mathbb{R}} r^{2}\left|w_{0}^{\prime}(r t)\right| \mathrm{dt}=r \int_{\mathbb{R}}\left|w_{0}^{\prime}(\sigma)\right| d \sigma$ and $\int r w_{0}(r \sigma) d \sigma=1$, we have

$$
\begin{aligned}
\forall \alpha \in\left\{x_{1}, \ldots, x_{d-1}, t\right\}, & \int_{\mathbb{R}^{d} \times \mathbb{R}^{+}} \partial_{\alpha}|w(x, t)| \mathrm{dx} \mathrm{dt} \leq r \int_{\mathbb{R}}\left|w_{0}^{\prime}(\sigma)\right| d \sigma \quad \text { and } \\
& \int_{\mathbb{R}^{d} \times \mathbb{R}^{+}} \partial_{x_{d}}|w(x, t)| \mathrm{dx} \mathrm{dt} \leq r_{d} \int_{\mathbb{R}}\left|w_{0}^{\prime}(\sigma)\right| d \sigma .
\end{aligned}
$$

We will make mechanical use of these estimates in the following proofs. We will also frequently and without specification use the inequality

$$
\int_{\Omega} \int_{\mathbb{R}^{+}} \psi(y, s) \lambda(y) \text { dy ds } \leq T|\Omega|\|\psi \lambda\|_{L^{\infty}\left(\mathbb{R}^{d} \times \mathbb{R}^{+}\right)} .
$$

Definition 5.6 (The form $\widetilde{E}^{+}$and the dual forms $E^{+*}$ and $\widetilde{E}^{+*}$ ). Let $c$ be the entropy solution of (1)-(3), and $c_{h}$ the discrete solution defined by (18). We define $\widetilde{E}^{+}\left(c_{h}, c, \psi \lambda\right)$ by

$$
\widetilde{E}^{+}\left(c_{h}, c, \psi \lambda\right):=\int_{\Omega \times \mathbb{R}^{+}} E^{+}\left(c_{h}, c(y, s), \varphi(\cdot, \cdot, y, s)\right) \mathrm{dy} \mathrm{ds},
$$

where $\varphi(x, t, y, s)$ is defined by (25) and, corresponding to the forms $E^{+}$and $\widetilde{E}^{+}$, we set

$$
\begin{aligned}
E^{+*}(\kappa, c, \psi \lambda):= & \int_{\Omega \times \mathbb{R}^{+}}(c(y, s)-\kappa)^{-} \partial_{s} \tilde{\varphi}(y, s) \mathrm{dy} \mathrm{ds} \\
& +\int_{\Omega \times \mathbb{R}^{+}} \Phi^{-}(c(y, s), \kappa) \cdot \nabla_{y} \tilde{\varphi}(y, s) \mathrm{dy} \mathrm{ds} \\
& +\int_{\Omega}\left(c_{0}(y)-\kappa\right)^{-} \tilde{\varphi}(y, 0) \mathrm{dy} \\
& -\int_{\partial \Omega \times(0, T)} \Phi^{-}(\gamma c(y, s), \kappa) \cdot \mathbf{n}(y, s) \widetilde{\varphi}(y, s) d \gamma(y) \mathrm{ds}, \\
\widetilde{E}^{+*}\left(c_{h}, c, \psi \lambda\right):= & \int_{\Omega \times \mathbb{R}^{+}} E^{+*}\left(c_{h}(x, t), c, \widetilde{\varphi}\right) \mathrm{dx} \mathrm{dt}
\end{aligned}
$$

with $\tilde{\varphi}(y, s)=\varphi(\cdot, \cdot, y, s)$. Here we denote by $\gamma c$ the trace of $c$ on the boundary $\partial \Omega \times(0, T)$, a function which is well defined (measurable and bounded a.e.) since $c \in B V \cap L^{\infty}(\Omega \times(0, T))$ (see Theorem 2.4).

The proof of the Propositions 5.1 and 5.2 falls into three parts. In the first part, we derive estimates on the quantity $\widetilde{E}^{+}\left(c_{h}, c, \psi \lambda\right)+\widetilde{E}^{+*}\left(c_{h}, c, \psi \lambda\right)$. In the second part, we analyze and give estimates on terms related to the behavior of $c$ and $c_{h}$ on the boundaries $\{t=0\}$ and $\partial \Omega \times(0, T)$ to deduce, in the third part, the estimates (5.1) and (24). 


\subsection{First step.}

Lemma 5.7. Let $c_{h}$ be the discrete solution defined by (18), and let $c$ be the entropy solution of (11)-(3). Then, under the Assumptions 3.1, 3.2, and 3.5, we have

$$
\begin{aligned}
& \widetilde{E}^{+}\left(c_{h}, c, \psi \lambda\right)+\widetilde{E}^{+*}\left(c_{h}, c, \psi \lambda\right) \\
& \quad \geq-\|\psi \lambda\|_{L^{\infty}\left(\mathbb{R}^{d} \times \mathbb{R}^{+}\right)}\left(\eta_{0}+\eta_{t}\left[K_{1} r\right]+\eta_{c}\left[K_{1} r+K_{1}^{\prime}\left(r+r_{d}\right)\right]+\bar{\eta}\right),
\end{aligned}
$$

where $\eta_{0}, \eta_{t}, \eta_{c}, \bar{\eta}$ are defined in Proposition [5.1.

Sketch of the proof. By the definition of entropy solution and by (8), we have $E^{+*}(\kappa, c, \psi) \geq 0$ for all $\kappa \in\left[C_{m}, C_{M}\right]$ and, hence, $\widetilde{E}^{+*}\left(c_{h}, c, \psi\right) \geq 0$. The lower estimate of $\widetilde{E}^{+}\left(c_{h}, c, \psi\right)$ relies on

(1) the approximate entropy inequality described in Lemma 4.5, and

(2) estimates on the $L^{\infty}$ norm of the function $(x, t) \mapsto \int_{\Omega \times \mathbb{R}^{+}} \varphi(x, t, y, s)$ dy ds defined by (25).

These last estimates make use of estimates on the $L^{1}$ norm of the approximation of the unit $w$, which are given by

$$
\begin{aligned}
\int_{\mathbb{R}^{d} \times \mathbb{R}^{+}} w(x-y, t-s) \mathrm{dxdt} & \leq 1, \quad \forall(x, t) \in \mathbb{R}^{d} \times \mathbb{R}^{+}, \\
\int_{\mathbb{R}^{d} \times \mathbb{R}^{+}}|\nabla w(x-y, t-s)| \mathrm{dxdt} & \leq\left(r+r_{d}\right) K_{1}^{\prime}, \quad \forall(x, t) \in \mathbb{R}^{d} \times \mathbb{R}^{+}, \\
\int_{\mathbb{R}^{d} \times \mathbb{R}^{+}}\left|\partial_{t} w(x-y, t-s)\right| \mathrm{dxdt} & \leq r K_{1}, \quad \forall(x, t) \in \mathbb{R}^{d} \times \mathbb{R}^{+},
\end{aligned}
$$

where $K_{1}, K_{1}^{\prime}$ are defined by

$$
K_{1}:=\int_{\mathbb{R}}\left|\partial_{t} w_{0}(t)\right| \mathrm{dt}, \quad K_{1}^{\prime}:=\int_{\mathbb{R}^{d}}\left|\nabla w_{0}\left(x_{1}\right) \cdots w_{0}\left(x_{d}\right)\right| \mathrm{dx} .
$$

5.2. Second step. The relation of symmetry $(s-\sigma)^{+}=(\sigma-s)^{-}$, applied with $s=c_{h}$ and $\sigma=c$, together with the identities $\left(\partial_{t}+\partial_{s}\right) w(x-y, t-s)=0$ and $\left(\nabla_{x}+\nabla_{y}\right) w(x-y, t-s)=0$ leads to

$$
\begin{aligned}
& \widetilde{E}^{+}\left(c_{h}, c, \varphi\right)+\widetilde{E}^{+*}\left(c_{h}, c, \bar{\varphi}\right) \\
& (31)=\int_{\Omega \times \mathbb{R}^{+}} \int_{\Omega \times \mathbb{R}^{+}}\left[\begin{array}{l}
\left(c_{h}(x, t)-c(y, s)\right)^{+} \psi_{s} \lambda(y) \\
+\Phi^{+}\left(c_{h}(x, t), c(y, s)\right) \cdot \nabla_{y}(\psi \lambda)
\end{array}\right] w(x-y, t-s) \mathrm{dx} \mathrm{dt} \mathrm{dy} \mathrm{ds}
\end{aligned}
$$$$
(32)+\int_{\Omega \times \mathbb{R}^{+}} \int_{\Omega}\left(c_{0}(x)-c(y, s)\right)^{+} \varphi(x, 0, y, s) \mathrm{dx} \mathrm{dt} \mathrm{dy}
$$

$$
-\int_{\Omega \times \mathbb{R}^{+}} \int_{\partial \Omega \times(0, T)} \Phi^{-}\left(\gamma c(y, s), c_{h}(x, t)\right) \varphi(x, t, y, s) \mathrm{dxdt} \mathrm{d} \gamma(y) \mathrm{ds} .
$$

Our aim is to estimate the term (31). In view of Lemma 5.7, this requires estimates on the terms (32) and (33), respectively, related to the behavior of the discrete and the entropy solution at initial time and at the boundary of the domain.

The term (32) is small with respect to $1 / r+1 / r_{d}$ because $c(y, 0+)=c_{0}(y)$ (the initial trace of the entropy solution coincides everywhere with the initial datum CH99a, EGGH98. This is no longer true on the boundary of the domain $\partial \Omega \times(0, T)$. The trace of the entropy solution may be distinct from the boundary datum on a part of the boundary. Therefore, in order to estimate the term (33) 
we have to use a specific technique. For that purpose, we introduce some functions of localization in order to give an accurate parametrization of the boundary. The supports of such functions, in particular, are chosen in order to isolate flat parts of the boundary of $\Omega$ (parts of the boundary of $\Omega$ which are included in a hyperplane of $\mathbb{R}^{d}$; recall that $\Omega$ is a convex polygonal open subset of $\mathbb{R}^{d}$ ).

Definition 5.8 (Localization). Let $\lambda$ be a function with values in $[0,1]$ such that $\operatorname{supp}(\lambda) \cap \partial \Omega$ is included in a hyperplane of $\mathbb{R}^{d}$, and such that the orthogonal projection of $\operatorname{supp}(\lambda) \cap \Omega$ on this hyperplane is included in $\operatorname{supp}(\lambda) \cap \partial \Omega$. Upon rotating and relabeling the axes (via the action of an orthogonal matrix $A \in \mathcal{O}_{d}(\mathbb{R})$ ), we can suppose that

$$
\operatorname{supp}(\lambda) \cap \Omega \subset \mathbb{R}_{+}^{d} \quad \text { and } \quad \operatorname{supp}(\lambda) \cap \partial \Omega \subset \mathbb{R}^{d-1} \times\{0\}=\mathbb{R}^{d-1},
$$

where $\mathbb{R}_{+}^{d}=\left\{x=\left(\bar{x}, x_{d}\right) \in \mathbb{R}^{d}, x_{d}>0\right\}$.

Up to now, we set $\lambda$ to represent this very function of localization in the definition of the mapping

$$
\varphi:(x, t, y, s) \longmapsto \psi(y, s) \lambda(y) w(x-y, t-s) .
$$

Besides, we will suppose that $r \leq r_{d}$, and that $r, r_{d}$ are large enough to ensure that (34) still holds when $K_{\lambda}=\operatorname{supp}(\lambda)$ is replaced by its neighborhood

$$
V_{r, r_{d}}\left(K_{\lambda}\right)=\left\{x \in \mathbb{R}^{d}, \operatorname{dist}\left(x, K_{\lambda}\right)<\sqrt{1 / r^{2}+1 / r_{d}^{2}}\right\} .
$$

To estimate the term (32), we set

$$
(y, s) \mapsto \int_{-\infty}^{-s} \varphi(x, 0, y,-\sigma) d \sigma
$$

and $\kappa=u_{0}(x)$ to be, respectively, the test-function and the parameter in the entropy inequality (6) (with negative semi-entropy) satisfied by $c$, and we integrate the result with respect to $x \in \Omega$. This yields

$$
\int_{\Omega \times \mathbb{R}^{+}} \int_{\Omega}\left(c_{0}(x)-c(y, s)\right)^{+} \varphi(x, 0, y, s) \mathrm{dx} \mathrm{dt} \mathrm{dy} \leq R_{1}+R_{2}+R_{3},
$$

where

$$
\begin{aligned}
R_{1} & =\int_{\Omega} \int_{\Omega \times \mathbb{R}^{+}} \Phi^{+}\left(c_{0}(x), c(y, s)\right) \cdot \nabla_{y}\left(\int_{-\infty}^{-s} \varphi(x, 0, y,-\sigma) d \sigma\right) \mathrm{dy} \mathrm{ds} \mathrm{dx}, \\
R_{2} & =\int_{\Omega} \int_{\Omega}\left(c_{0}(x)-c_{0}(y)\right)^{+} \psi(y, s) \lambda(y) \tilde{w}(x-y) \mathrm{dy} \mathrm{dx}, \\
R_{3} & =\mathcal{L} \int_{\Omega} \int_{\partial \Omega \times \mathbb{R}^{+}}\left(c_{0}(x)-\bar{c}(y, s)\right)^{+} \int_{-\infty}^{-s} \varphi(x, 0, y,-\sigma) d \sigma d \gamma(y) \mathrm{ds} \mathrm{dx} .
\end{aligned}
$$

Here $\tilde{w}$ denotes the approximation of the unit $x \mapsto r w_{0}\left(r x_{1}\right) \cdots r w_{0}\left(x_{d-1}\right) r_{d} w_{0}\left(x_{d}\right)$.

Since

$$
\nabla_{y}\left(\int_{-\infty}^{-s} \varphi(x, 0, y,-\sigma) d \sigma\right)=-\nabla_{x}\left(\int_{-\infty}^{-s} \varphi(x, 0, y,-\sigma) d \sigma\right),
$$

we have

$$
R_{1}=-\int_{\Omega} \int_{\Omega \times \mathbb{R}^{+}} \Phi^{+}\left(c_{0}(x), c(y, s)\right) \cdot \nabla_{x}\left(\int_{-\infty}^{-s} \varphi(x, 0, y,-\sigma) d \sigma\right) \mathrm{dy} \mathrm{ds} \mathrm{dx} .
$$


Besides, for $x \in \partial \Omega \cap V_{r, r_{d}}\left(K_{\lambda}\right), y \in \Omega \cap K_{\lambda}$, we have, by (34), $(x-y)_{d} \leq 0$ and, since $\operatorname{supp}\left(w_{0}\right) \subset[0,1], \varphi(x, 0, y,-\sigma)=0$. In particular, by integrating by parts with respect to $x$, we have that

$$
\int_{\Omega} \int_{\Omega \times \mathbb{R}^{+}} \Phi^{+}\left(c_{0}(y), c(y, s)\right) \cdot \nabla_{x}\left(\int_{-\infty}^{-s} \varphi(x, 0, y,-\sigma) d \sigma\right) \mathrm{dy} \mathrm{ds} \mathrm{dx}=0
$$

and

$$
\begin{aligned}
R_{1}= & \int_{\Omega} \int_{\Omega \times \mathbb{R}^{+}}\left(\Phi^{+}\left(c_{0}(y), c(y, s)\right)-\Phi^{+}\left(c_{0}(x), c(y, s)\right)\right) \\
& \cdot \nabla_{x}\left(\int_{-\infty}^{-s} \varphi(x, 0, y,-\sigma) d \sigma\right) \mathrm{dy} \mathrm{ds} \mathrm{dx} .
\end{aligned}
$$

Since

$$
\left|\left(\Phi^{+}\left(c_{0}(y), c(y, s)\right)-\Phi^{+}\left(c_{0}(x), c(y, s)\right)\right)\right| \leq \mathcal{L}\left|c_{0}(y)-c_{0}(x)\right| \leq \mathcal{L}\left|c_{0}\right|_{B V}|x-y|,
$$

we have

$$
\begin{aligned}
R_{1} \leq & \mathcal{L}\left|c_{0}\right|_{B V}\|\psi \lambda\|_{L^{\infty}\left(\mathbb{R}^{d} \times \mathbb{R}^{+}\right)} \int_{\Omega} \int_{\Omega}|x-y|\left|\nabla_{x} \tilde{w}(x-y)\right| \mathrm{dy} \mathrm{dx} \\
& \times\left(\int_{\mathbb{R}^{+}} \int_{-\infty}^{-s} r w_{0}(-r \sigma) d \sigma \mathrm{ds}\right) .
\end{aligned}
$$

The inequality

$$
\int_{\mathbb{R}^{+}} \int_{-\infty}^{-s} r w_{0}(-r \sigma) d \sigma \mathrm{ds}=\frac{1}{r} \int_{0}^{1} \sigma w_{0}(\sigma) d \sigma \leq \frac{1}{r} \int_{0}^{1} w_{0}(\sigma) d \sigma=\frac{1}{r}
$$

gives

$$
R_{1} \leq \mathcal{L}\left|c_{0}\right|_{B V}\|\psi \lambda\|_{L^{\infty}\left(\mathbb{R}^{d} \times \mathbb{R}^{+}\right)} \int_{\Omega} \int_{\Omega}|x-y|\left|\nabla_{x} \tilde{w}(x-y)\right| \mathrm{dy} \mathrm{dx} \frac{1}{r} .
$$

Furthermore, by (26) we have

$$
\begin{aligned}
\int_{\Omega} \int_{\Omega}|x-y|\left|\nabla_{x} \tilde{w}(x-y)\right| \mathrm{dy} \mathrm{dx} & =\int_{\Omega} \int_{x-\Omega}|z||\nabla \tilde{w}(z)| \mathrm{dzdx} \\
\leq|\Omega| \int_{\mathbb{R}^{d}}|z||\nabla \tilde{w}(z)| \mathrm{dz} & \leq|\Omega| K_{1}^{\prime}\left(d-1+\frac{r}{r_{d}}\right), \quad \text { since } r \leq r_{d} .
\end{aligned}
$$

Eventually, we have

$$
\begin{aligned}
R_{1} & \leq \mathcal{L}\left|c_{0}\right|_{B V}|\Omega| K_{1}^{\prime}(d-1)\|\psi \lambda\|_{L^{\infty}\left(\mathbb{R}^{d} \times \mathbb{R}^{+}\right)}\left(\frac{1}{r}+\frac{1}{r_{d}}\right) \\
& \leq K_{0}\|\psi \lambda\|_{L^{\infty}\left(\mathbb{R}^{d} \times \mathbb{R}^{+}\right)} \frac{1}{r},
\end{aligned}
$$

where the constant $K_{0}$ is defined by (23).

Similar computations (see [CH99a, [EGGH98]) lead to the estimates $R_{2}, R_{3} \leq$ $K_{0} \frac{1}{r}\|\psi \lambda\|_{L^{\infty}\left(\mathbb{R}^{d} \times \mathbb{R}^{+}\right)}$, and we have

(38) $\int_{\Omega \times \mathbb{R}^{+}} \int_{\Omega}\left(c_{0}(x)-c(y, s)\right)^{+} \varphi(x, 0, y, s) \mathrm{dx} \mathrm{dt} \mathrm{dy} \leq 3 K_{0}\|\psi \lambda\|_{L^{\infty}\left(\mathbb{R}^{d} \times \mathbb{R}^{+}\right)} \frac{1}{r}$. 
We now prove the following estimate on (33):

$$
\begin{aligned}
\bar{I}_{\lambda}:=- & \int_{\Omega \times \mathbb{R}^{+}} \int_{\partial \Omega \times(0, T)} \Phi^{-}\left(\gamma c(y, s), c_{h}(x, t)\right) \cdot \mathbf{n}(y) \varphi(x, t, y, s) \mathrm{dx} \mathrm{dt} \mathrm{d} \gamma(y) \mathrm{ds} \\
& \leq K_{0}\|\psi \lambda\|_{L^{\infty}\left(\mathbb{R}^{d} \times \mathbb{R}_{+}\right)}(1+2 r) \frac{1}{r_{d}}+\|\psi \lambda\|_{L^{\infty}\left(\mathbb{R}^{d} \times \mathbb{R}^{+}\right)}\left(\eta_{0}+\eta_{t}+\eta_{c}+\bar{\eta}\right) .
\end{aligned}
$$

The identity $(c-\kappa)^{-}=(c \top w-\kappa)^{-}+(c-\kappa \top w)^{-}$holds for every $c, \kappa, w \in \mathbb{R}$. As a counterpart the corresponding entropy fluxes satisfy the relation

$$
\Phi^{-}(c, \kappa)=\Phi^{-}(c \top w, \kappa)+\Phi^{-}(c, \kappa \perp w)
$$

and, for a.e. $(x, t, y, s) \in \Omega \times(0, T) \times \partial \Omega \times(0, T)$, we have

$$
\begin{aligned}
-\Phi^{-}\left(\gamma c(y, s), c_{h}(x, t)\right) \cdot \mathbf{n}(y)= & -\Phi^{-}\left(\gamma c(y, s) \top \bar{c}(y, s), c_{h}(x, t)\right) \cdot \mathbf{n}(y) \\
& -\Phi^{-}\left(\gamma c(y, s), c_{h}(x, t) \perp \bar{c}(y, s)\right) \cdot \mathbf{n}(y) .
\end{aligned}
$$

By (10), we have

$$
-\Phi^{-}\left(\gamma c(y, s), c_{h}(x, t) \perp \bar{c}(y, s)\right) \cdot \mathbf{n}(y) \leq \mathcal{L}\left(\bar{c}(y, s)-c_{h}(x, t) \perp \bar{c}(y, s)\right)^{-}=0,
$$

and therefore $\bar{I}_{\lambda} \leq \bar{J}_{\lambda}$ with

$$
\begin{aligned}
\bar{J}_{\lambda}:=-\int_{\Omega \times \mathbb{R}^{+}} \int_{\partial \Omega \times(0, T)} \Phi^{-}\left(\gamma c(y, s) \top \bar{c}(y, s), c_{h}(x, t)\right) \\
\cdot \mathbf{n}(y) \varphi_{A_{\lambda}}(x, t, y, s) \mathrm{dx} \mathrm{dt} \mathrm{d} \gamma(y) \mathrm{ds} .
\end{aligned}
$$

From the relation of symmetry $\Phi^{-}(a, b)=\Phi^{+}(b, a)$ it follows that

$$
\begin{array}{r}
\bar{J}_{\lambda}=-\int_{\mathbb{R}_{+}^{d} \times \mathbb{R}_{+}} \int_{0}^{T} \int_{\mathbb{R}^{d-1}} \Phi^{+}\left(c_{h}(x, t), \gamma c(\bar{y}, s) \top \bar{c}(\bar{y}, s)\right) \\
\cdot \mathbf{n}(y) \lambda(y) \varphi(x, t, \bar{y}, s) \mathrm{dx} \mathrm{dt} \mathrm{d} \bar{y} \mathrm{ds} .
\end{array}
$$

We now make use of the approximate entropy inequality (22) satisfied by $c_{h}$ to get an estimate on $\bar{J}_{\lambda}$. Choose $\kappa:=\gamma c \top \bar{c}(\bar{y}, s)$, and the test function $(x, t) \mapsto \varphi^{\star}(x, t)$ in (22), where

$$
\begin{aligned}
\varphi^{\star}(x, t):= & \lambda(y) \psi(\bar{y}, 0, s) r w_{0}\left(r\left(x_{1}-y_{1}\right)\right) \cdots w_{0}\left(r\left(x_{d-1}-y_{d-1}\right)\right) \\
& \left(\int_{x_{d}}^{+\infty} r_{d} w_{0}\left(r_{d} \sigma\right) d \sigma\right) r w_{0}(r(t-s)) .
\end{aligned}
$$

Integrate the result with respect to $(\bar{y}, s)$. This yields

$$
\int_{\mathbb{R}_{+}^{d} \times \mathbb{R}_{+}} E^{+}\left(c_{h}, \gamma c \top \bar{c}(\bar{y}, s), \varphi^{\star}\right) d \bar{y} \mathrm{ds} \geq F,
$$

where the right hand side $F$ can be estimated from below by

$$
F \geq-\|\psi \lambda\|_{L^{\infty}\left(\mathbb{R}^{d} \times \mathbb{R}^{+}\right)}\left(\eta_{0}+\eta_{t}\left[K_{1} r\right]+\eta_{c}\left[K_{1} r+K_{1}^{\prime}\left(r+r_{d}\right)\right]+\bar{\eta}\right),
$$

exactly as in the proof of Lemma 5.7, leading to

$$
\begin{aligned}
& \int_{\mathbb{R}_{+}^{d} \times \mathbb{R}_{+}} E^{+}\left(c_{h}, \gamma c \top \bar{c}(\bar{y}, s), \varphi^{\star}\right) d \bar{y} \mathrm{ds} \\
& \quad \geq-\|\psi \lambda\|_{L^{\infty}\left(\mathbb{R}^{d} \times \mathbb{R}^{+}\right)}\left(\eta_{0}+\eta_{t}\left[K_{1} r\right]+\eta_{c}\left[K_{1} r+K_{1}^{\prime}\left(r+r_{d}\right)\right]+\bar{\eta}\right) .
\end{aligned}
$$


On the other hand, we have

$$
\int_{\mathbb{R}_{+}^{d} \times \mathbb{R}_{+}} E^{+}\left(c_{h}, \gamma c(\bar{y}, s) \top \bar{c}(\bar{y}, s), \varphi^{\star}\right) d \bar{y} \mathrm{ds}=A_{1}+A_{2}+A_{3}+A_{4},
$$

where

$$
\begin{aligned}
& A_{1}=\int_{\mathbb{R}^{d-1} \times \mathbb{R}_{+}} \int_{\mathbb{R}_{+}^{d} \times \mathbb{R}_{+}}\left(c_{h}-\gamma c \top \bar{c}(\bar{y}, s)\right)^{+} \partial_{t} \varphi^{\star} \mathrm{dxdt} \mathrm{d} \bar{y} \mathrm{ds}, \\
& A_{2}=\int_{\mathbb{R}^{d-1} \times \mathbb{R}_{+}} \int_{\mathbb{R}_{+}^{d} \times \mathbb{R}_{+}} \Phi^{+}\left(\left(c_{h}, \gamma c \top \bar{c}(\bar{y}, s)\right) \cdot \nabla \varphi^{\star} \mathrm{dxdt} \mathrm{d} \bar{y} \mathrm{ds},\right. \\
& A_{3}=\int_{\mathbb{R}^{d-1} \times \mathbb{R}_{+}} \int_{\mathbb{R}_{+}^{d}}\left(c_{0}(x)-\gamma c \top \bar{c}(\bar{y}, s)\right)^{+} \varphi^{\star}(0, x, s, \bar{y}) \mathrm{dxd} \mathrm{d} \mathrm{ds}, \\
& A_{4}=\mathcal{L} \int_{\mathbb{R}^{d-1} \times \mathbb{R}_{+}} \int_{\mathbb{R}^{d-1} \times \mathbb{R}_{+}}(\bar{c}(\bar{x}, 0)-\gamma c \top \bar{c}(\bar{y}, s))^{+} \varphi^{\star}(t, \bar{x}, 0, s, \bar{y}) \mathrm{d} \overline{\mathrm{x}} \mathrm{dt} \mathrm{d} \bar{y} \mathrm{ds} .
\end{aligned}
$$

Since $\operatorname{supp}\left(w_{0}\right) \subset[0,1]$, we have $A_{3}=0$. Besides, the terms $A_{1}, A_{4}$ are small with respect to $1 / r_{d}$. Indeed, we have

$$
\int_{0}^{\infty} \int_{x_{d}}^{\infty} r_{d} w_{0}\left(r_{d} \sigma\right) d \sigma d x_{d}=\frac{1}{r_{d}} \int_{0}^{1} \sigma w_{0}(\sigma) d \sigma \leq \frac{1}{r_{d}}
$$

and, by (26), (27),

$$
\left|A_{1}\right| \leq 2 \mathcal{C}\left\|w_{0}^{\prime}\right\|_{L^{1}(\mathbb{R})} T|\Omega|\|\psi \lambda\|_{L^{\infty}\left(\mathbb{R}^{d} \times \mathbb{R}_{+}\right)} \frac{r}{r_{d}} \leq K_{0}\|\psi \lambda\|_{L^{\infty}\left(\mathbb{R}^{d} \times \mathbb{R}_{+}\right)} \frac{r}{r_{d}} .
$$

Besides, we have $(\bar{c}(\bar{x}, t)-\gamma c \top \bar{c}(\bar{y}, s))^{+} \leq(\bar{c}(\bar{x}, t)-\bar{c}(\bar{y}, s))^{+}$, and, therefore,

$$
\left|A_{4}\right| \leq\left.|\bar{c}|_{B V} T|\Omega||| \psi \lambda\left\|_{L^{\infty}\left(\mathbb{R}^{d} \times \mathbb{R}_{+}\right)} \frac{1}{r} \leq K_{0}\right\| \psi \lambda\right|_{L^{\infty}\left(\mathbb{R}^{d} \times \mathbb{R}_{+}\right)} \frac{1}{r}
$$

We now intend to compare $-A_{2}$ to $\bar{J}_{\lambda}$. Since $\partial_{x_{d}} \varphi^{\star}(x, t, \bar{y}, s)=\lambda(y) \varphi(x, t, \bar{y}, s)$ and since $\mathbf{n}(y)=(0, \ldots, 0,-1)^{T} \in \mathbb{R}^{d}$, we have, by (40),

$$
A_{2}+\bar{J}_{\lambda}=\int_{\mathbb{R}^{d-1} \times \mathbb{R}_{+}} \int_{\mathbb{R}_{+}^{d} \times \mathbb{R}_{+}} \Phi^{+}\left(\left(c_{h}, \gamma c \top \bar{c}(\bar{y}, s)\right) \cdot \bar{\nabla} \varphi^{\star} \mathrm{dx} \mathrm{dt} \mathrm{d} \bar{y} \mathrm{ds},\right.
$$

where $\bar{\nabla} \varphi^{\star}=\left(\partial_{x_{1}} \varphi^{\star}, \ldots, \partial_{x_{d-1}} \varphi^{\star}, 0\right)^{T} \in \mathbb{R}^{d}$. By (43) we have

$$
\begin{aligned}
\left|A_{2}+\bar{J}_{\lambda}\right| & \leq 2 \mathcal{L C}(d-1)\left\|w_{0}^{\prime}\right\|_{L^{1}(\mathbb{R})} T|\Omega|\|\psi \lambda\|_{L^{\infty}\left(\mathbb{R}^{d} \times \mathbb{R}_{+}\right)} \frac{r}{r_{d}} \\
& \leq K_{0}\|\psi \lambda\|_{L^{\infty}\left(\mathbb{R}^{d} \times \mathbb{R}_{+}\right)} \frac{r}{r_{d}} .
\end{aligned}
$$

From (41), (42), (44), (45), and (46) follows (39).

5.3. Third step. Set $\psi(s, y)=\psi(s)=\frac{T-s}{T} \chi_{(0, T)}(s)$. Then $0 \leq \psi \leq 1$ and, collecting (29), (31)-(33), (38), and (39), we have

$$
\begin{aligned}
& \int_{\Omega \times(0, T)} \int_{\Omega \times(0, T)}\left[\begin{array}{c}
\frac{-1}{T}\left(c_{h}(x, t)-c(y, s)\right)^{+} \lambda(y) \\
+\Phi^{+}\left(c_{h}(x, t), c(y, s)\right) \cdot \nabla_{y} \lambda
\end{array}\right] w(x-y, t-s) \mathrm{dx} \mathrm{dt} \mathrm{dy} \mathrm{ds} \\
& \geq-\|\lambda\|_{L^{\infty}\left(\mathbb{R}^{d}\right)}\left(2\left(\eta_{0}+\eta_{t}\left[K_{1} r\right]+\eta_{c}\left[K_{1} r+K_{1}^{\prime}\left(r+r_{d}\right)\right]+\bar{\eta}\right)+K_{0}\left(\frac{4}{r}+2 \frac{r}{r_{d}}\right)\right),
\end{aligned}
$$


where $\lambda$ is defined in Definition 5.8 . Since $0 \leq \lambda \leq 1$, we have in particular

$$
\begin{aligned}
& \int_{\Omega \times(0, T)} \int_{\Omega \times(0, T)}\left[\begin{array}{c}
\frac{-1}{T}\left(c_{h}(x, t)-c(y, s)\right)^{+} \lambda(y) \\
+\Phi^{+}\left(c_{h}(x, t), c(y, s)\right) \cdot \nabla_{y} \lambda
\end{array}\right] w(x-y, t-s) \mathrm{dx} d t \mathrm{dy} \mathrm{ds} \\
& \quad \geq-\left(2\left(\eta_{0}+\eta_{t}\left[K_{1} r\right]+\eta_{c}\left[K_{1} r+K_{1}^{\prime}\left(r+r_{d}\right)\right]+\bar{\eta}\right)+K_{0}\left(\frac{4}{r}+2 \frac{r}{r_{d}}\right)\right) .
\end{aligned}
$$

We gather those local estimates (47) to get a global estimate. Denote by $\left(A_{i}\right)_{i=1, \ldots, N_{f}}$ the faces of $\Omega$, and by $n_{i}$ the outward unit normal to $\Omega$ along $A_{i}$. Let $B_{i}^{\tilde{r}}$ be the subset of all $x \in \Omega$ such that $\operatorname{dist}\left(x, A_{i}\right)<\frac{1}{\tilde{r}}$ and $\operatorname{dist}\left(x, A_{i}\right)<\operatorname{dist}\left(x, A_{j}\right)$ if $i \neq j$; define $G_{i}^{\tilde{r}}$ to be the largest cylinder generated by $n_{i}$ included in $B_{i}^{\tilde{r}}$, and set $\Delta_{i}^{\tilde{r}}=B_{i}^{\tilde{r}} \backslash G_{i}^{\tilde{r}}, \Omega_{\tilde{r}}=\Omega \backslash\left(\bigcup_{1, N_{f}} \Delta_{i}^{\tilde{r}}\right)$ and $b^{\tilde{r}}=\mathbb{1}_{\Omega_{\tilde{r} / 2}} \star \rho_{\tilde{r} / 4}$.

An estimate as (47) remains true if $\lambda=\lambda_{0}$, where $\lambda_{0}$ is a localization function with support included in $\Omega$. Then no boundary conditions have to be taken into account, and the term (33) can be considered to be zero. Now, we can write $b^{\tilde{r}}=\sum_{i=0, N_{f}} \lambda_{i}$ for such a function $\lambda_{0}$ and for functions $\left(\lambda_{i}\right)_{1, N_{f}}$ satisfying the hypotheses of Definition 5.8. Therefore, we have that

$$
\begin{aligned}
& \iint_{(\Omega \times(0, T))^{2}} \frac{-1}{T}\left(c_{h}(x, t)-c(y, s)\right)^{+} w(x-y, t-s) \mathrm{dxdt} \mathrm{dy} \mathrm{ds} \geq \delta_{1}+\delta_{2} \\
& (48)-\left(N_{f}+1\right)\left(2\left(\eta_{0}+\eta_{t}\left[K_{1} r\right]+\eta_{c}\left[K_{1} r+K_{1}^{\prime}\left(r+r_{d}\right)\right]+\bar{\eta}\right)+K_{0}\left(\frac{4}{r}+2 \frac{r}{r_{d}}\right)\right),
\end{aligned}
$$

where

$$
\begin{aligned}
& \delta_{1}=\iint_{(\Omega \times(0, T))^{2}} \frac{-1}{T}\left(c_{h}(x, t)-c(y, s)\right)^{+}\left(1-\sum_{i=0, N_{f}} \lambda_{i}\right) w(x-y, t-s) \mathrm{dxd} \mathrm{dyds}, \\
& \delta_{2}=\iint_{(\Omega \times(0, T))^{2}} \Phi^{+}\left(c_{h}(x, t), c(y, s)\right) \cdot \nabla\left(1-\sum_{i=0, N_{f}} \lambda_{i}\right) w(x-y, t-s) \mathrm{dxdt} \mathrm{dyds} .
\end{aligned}
$$

We have $\left|\Omega \backslash \Omega_{\tilde{r}}\right| \leq \frac{C_{\Omega}}{\tilde{r}^{2}}$ where $C_{\Omega}$ is a constant which depends only on $\Omega$ and $\left\|\nabla \lambda_{i}\right\|_{L^{\infty}} \leq \tilde{r}$ so that, for $\tilde{r}<1$,

$$
\left|\delta_{1}\right| \leq 2 \mathcal{C} T|\Omega| \frac{C_{\Omega}}{\tilde{r}^{2}} \leq \frac{K_{0}}{\tilde{r}}, \quad\left|\delta_{2}\right| \leq 2 \mathcal{L C} T|\Omega| \frac{C_{\Omega}}{\tilde{r}} \leq \frac{K_{0}}{\tilde{r}} .
$$

Besides, by the $B V$ estimate (77), we have

$$
\begin{aligned}
& \iint_{(\Omega \times(0, T))^{2}}\left(c_{h}(x, t)-c(y, s)\right)^{+} w(x-y, t-s) \mathrm{dxdt} \mathrm{dyds}-\left\|\left(c_{h}-c\right)^{+}\right\|_{L^{1}(\Omega \times(0, T))} \\
& \quad=\iint_{(\Omega \times(0, T))^{2}}\left(\left(c_{h}(x, t)-c(y, s)\right)^{+}-\left(c_{h}(x, t)-c(x, t)\right)^{+}\right) w(x-y, t-s) \mathrm{dx} \mathrm{dt} \mathrm{dy} \mathrm{ds} \\
& \geq-\iint_{(\Omega \times(0, T))^{2}}|c(y, s)-c(x, t)| w(x-y, t-s) \mathrm{dxdt} \mathrm{dy} \mathrm{ds} \\
& \geq-C_{B V} T|\Omega| \frac{1}{r} \geq-K_{0} \frac{1}{r}
\end{aligned}
$$


and, consequently,

$$
\begin{aligned}
&\left\|\left(c_{h}-c\right)^{+}\right\|_{L^{1}(\Omega \times(0, T))} \\
& \leq 2 T K_{0} \frac{1}{\tilde{r}} \\
&+2 T\left(N_{f}+1\right)\left[\eta_{0}+\bar{\eta}+\left(\eta_{t}+\eta_{c}\right) K_{1} r+\eta_{c} K_{1}^{\prime}\left(r+r_{d}\right)+K_{0}\left(\frac{2}{r}+\frac{r}{r_{d}}\right)\right] .
\end{aligned}
$$

We let $\tilde{r} \rightarrow+\infty$ in this last result and then minimize the right hand side with respect to $r$ and $r_{d}$ to get the a posteriori error estimate

$$
\begin{aligned}
&\left\|\left(c_{h}-c\right)^{+}\right\|_{L^{1}(\Omega \times(0, T))} \\
& \leq 2 T\left(N_{f}+1\right) \\
& \times \min _{r, r_{d} \in \mathbb{R}^{+}}\left[\eta_{0}+\bar{\eta}+\left(\eta_{t}+\eta_{c}\right) K_{1} r+\eta_{c} K_{1}^{\prime}\left(r+r_{d}\right)+K_{0}\left(\frac{2}{r}+1 \frac{r}{r_{d}}\right)\right] .
\end{aligned}
$$

The a priori error estimate now follows with the estimates on $\eta_{0}, \bar{\eta}, \eta_{t}, \eta_{c}$ (see CH99a, EGGH98), i.e.,

$$
\eta_{0}+\bar{\eta}+\eta_{t}+\eta_{c} \leq K h^{1 / 2}
$$

and choosing, for example,

$$
r:=\left(\frac{\left(\eta_{t}+\eta_{c}\right) K_{1}+\eta_{c} K_{1}^{\prime}}{K_{0}}\right)^{-1 / 3}, \quad r_{d}:=\left(\frac{K_{0} r}{\eta_{c} K_{1}^{\prime}}\right)^{1 / 2}
$$

Remark 5.9 (Nonoptimal order of convergence). The error estimates in Propositions 5.1 and 5.2 are nonoptimal compared to the convergence rate $h^{1 / 4}$ that can be proved for finite volume approximations of the Cauchy problem (cf. CH99a). The nonoptimality of our result comes from the estimate on the boundary term (39). Let us mention that in the special situation where $\mathbf{F}(x, t, c)=\mathbf{u}(x, t) f(c)$, and $f$ is monotone, this estimate can be improved, and the order $h^{1 / 4}$ is recovered (see also [Vig97]). However, the improvement in this special situation makes excessive use of the a priori knowledge of inflow and outflow boundaries and gives no hint to improve our general result.

\section{AdAPTIVE ALGORITHM AND NUMERICAL EXPERIMENTS}

In this subsection we will derive an adaptive algorithm from our theoretical a posteriori result in Proposition 5.1, and we will give some numerical experiments in order to demonstrate the applicability of the resulting adaptive solution scheme. In addition, we will demonstrate that the creation of artificial boundary layers depends on the choice of the numerical flux function.

6.1. Adaptive algorithm. The adaptive solution algorithm is derived by localizing the global error estimator $\eta$ of Proposition 5.1 into local error indicators $\eta_{k}^{n}$. Here $n$ denotes the time step and $k$ the triangle number of the underlying mesh. An equal distribution strategy of the local indicators leads us to the space adaptive algorithm. The adaptive time step is implicitly given through the CFL condition. As the derivation of the adaptive algorithm is a direct generalization of the algorithm on unbounded domains we refer to KO00, and Ohl01a, Ohl01b for further details. 

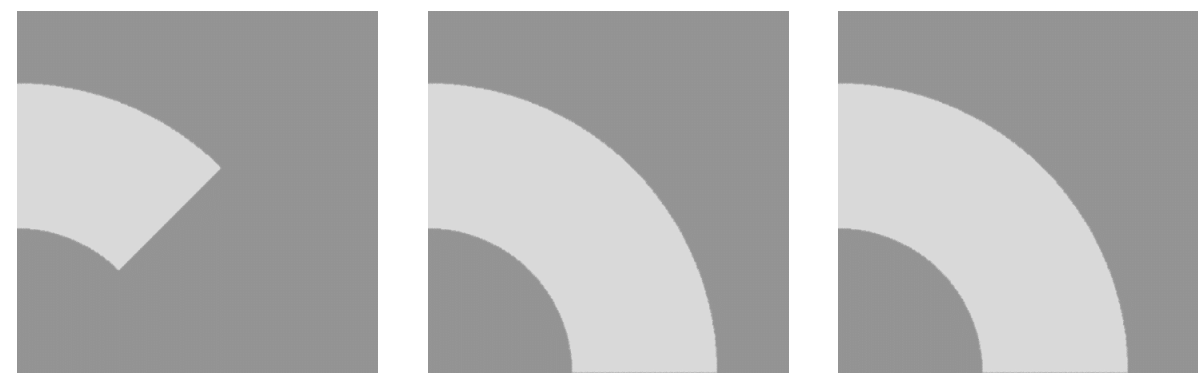

Figure 1. Color shading of the exact solution of the linear problem at $t=\pi / 4, t=\pi / 2$, and $t=2$.

6.2. First example: Linear transport problem. As a first example we choose a linear problem where the inflow and outflow regions are known a priori. The example is chosen, for instance, as it comes with a known exact solution. Thus, we can compare the $L^{1}$-error between the exact and the approximate solution with the error estimator $\eta$ defined in Proposition 5.1 .

We look at the following initial boundary value problem in $\Omega:=(0,1) \times(0,1)$ :

$$
\begin{aligned}
c_{t}+\nabla \cdot(\mathbf{u} c) & =0 \text { in } \Omega \times(0, T), \\
c(\cdot, 0) & =0 \text { in } \Omega, \\
c(x, t) & =\bar{c}(t, x) \text { in } \partial \Omega \times(0, T) .
\end{aligned}
$$

Then $c(x, t)$ is constant along the streamlines of the prescribed velocity field $\mathbf{u}\left(x_{1}, x_{2}\right):=\left(x_{2},-x_{1}\right)^{\top}$, and therefore only depends on the initial data and the boundary values at the inflow boundary. In our example we set

$$
\bar{c}(t, x):= \begin{cases}1, & \text { if } x \in\{0\} \times(0.4,0.8), \\ 0, & \text { else. }\end{cases}
$$

The exact solution (see Figure 1) of this problem is

$$
c(t, x):= \begin{cases}1, & \text { if } \arcsin \left(\frac{x_{1}}{|x|}\right)-t \leq 0, \text { and }|x| \in(0.4,0.8), \\ 0, & \text { else. }\end{cases}
$$
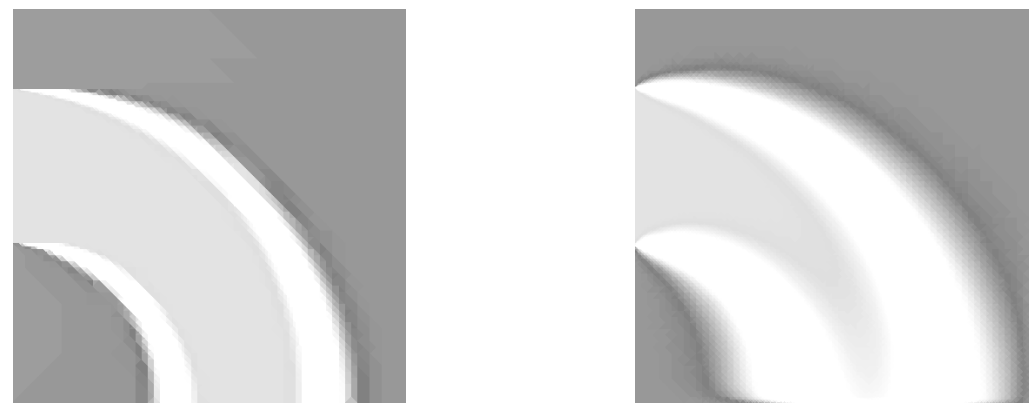

Figure 2. Comparison of the boundary layer behavior at the outflow boundary for the full upwind flux (left), and the LaxFriedrichs flux (right). 
TABLE 1. Comparison of the $L^{1}$-error and the convergence rate for upwind and Lax-Friedrichs flux on uniform meshes.

\begin{tabular}{|r||c|c||c|c|}
\hline $\begin{array}{r}\text { Number of } \\
\text { triangles }\end{array}$ & $\begin{array}{c}L^{1} \text {-error } \\
\text { Upwind flux }\end{array}$ & $\begin{array}{c}\text { Order of } \\
L^{1} \text {-error }\end{array}$ & $\begin{array}{c}L^{1} \text {-error } \\
\text { Lax-Friedrichs flux }\end{array}$ & $\begin{array}{c}\text { Order of } \\
L^{1} \text {-error }\end{array}$ \\
\hline \hline 4096 & $9.244172 \mathrm{E}-02$ & & $2.496511 \mathrm{E}-01$ & \\
\hline 16384 & $6.307752 \mathrm{E}-02$ & 0.551 & $1.845089 \mathrm{E}-01$ & 0.436 \\
\hline 65536 & $4.460084 \mathrm{E}-02$ & 0.500 & $1.342645 \mathrm{E}-01$ & 0.459 \\
\hline 262144 & $3.174344 \mathrm{E}-02$ & 0.491 & $9.676411 \mathrm{E}-02$ & 0.473 \\
\hline 1048576 & $2.252004 \mathrm{E}-02$ & 0.495 & $6.918218 \mathrm{E}-02$ & 0.484 \\
\hline
\end{tabular}

TABLE 2. Comparison of the error estimator $\eta$ and its convergence rate for upwind and Lax-Friedrichs flux on uniform meshes.

\begin{tabular}{|r||c|c||c|c|}
\hline $\begin{array}{r}\text { Number of } \\
\text { triangles }\end{array}$ & $\begin{array}{c}\eta \\
\text { Upwind flux }\end{array}$ & $\begin{array}{c}\text { Order of } \\
\eta\end{array}$ & $\begin{array}{c}\eta \\
\text { Lax-Friedrichs flux }\end{array}$ & $\begin{array}{c}\text { Order of } \\
\eta\end{array}$ \\
\hline \hline 4096 & 0.4140817 & & 0.7457754 & \\
\hline 16384 & 0.3256964 & 0.346 & 0.6108820 & 0.288 \\
\hline 65536 & 0.2594602 & 0.328 & 0.4922482 & 0.312 \\
\hline 262144 & 0.2070642 & 0.325 & 0.3922287 & 0.328 \\
\hline 1048576 & 0.1645522 & 0.332 & 0.3102630 & 0.338 \\
\hline
\end{tabular}

In our first numerical experiment we compare the generation of an artificial boundary layer at the outflow boundary for two different numerical flux functions. As Figure 2 clearly shows, an artificial boundary layer is created by using the Lax-Friedrich flux, while no artificial layer is produced with full upwinding (e.g., Engquist-Osher, or Godunov flux). For a detailed study of this boundary layer behavior in one space dimension, we refer to CHG01. In addition we remark that in the case of systems of conservation laws it might be necessary to choose a Godunov flux at the boundary in order to get a proper discretization of the boundary conditions. In the scalar case the creation of an artificial boundary layer does not influence the convergence rate of the scheme. For instance, in our example both methods converge with an experimental order of convergence of $h^{1 / 2}$ where $h$ denotes the uniform mesh size. Nevertheless, the absolute error for the upwind method is much smaller than in the Lax-Friedrichs case (see Table 1).

The influence of the choice of the numerical flux function on the adaptive solution algorithm is shown in Figure 3, While in both cases the interior layers are resolved by the adaptive algorithm, the grid is additionally refined in the artificial boundary layer in the case of the Lax-Friedrichs flux. This leads to some extra numerical cost which might lead to an inefficient adaptive numerical scheme.

In Table 2 we give the values of the error estimator $\eta$ of Proposition 5.1 The numerical order of convergence of the estimator is $h^{1 / 3}$ for both choices of the numerical flux (see Table 2). Our theoretical investigation would lead to the same order of convergence if we would be able to prove that the numerical solution is uniformly bounded in BV. Up to now such a bound is only available in one space dimension or for finite volume schemes on structured rectangular grids. In the general case we are only able to show that the BV norm of the approximate solution 

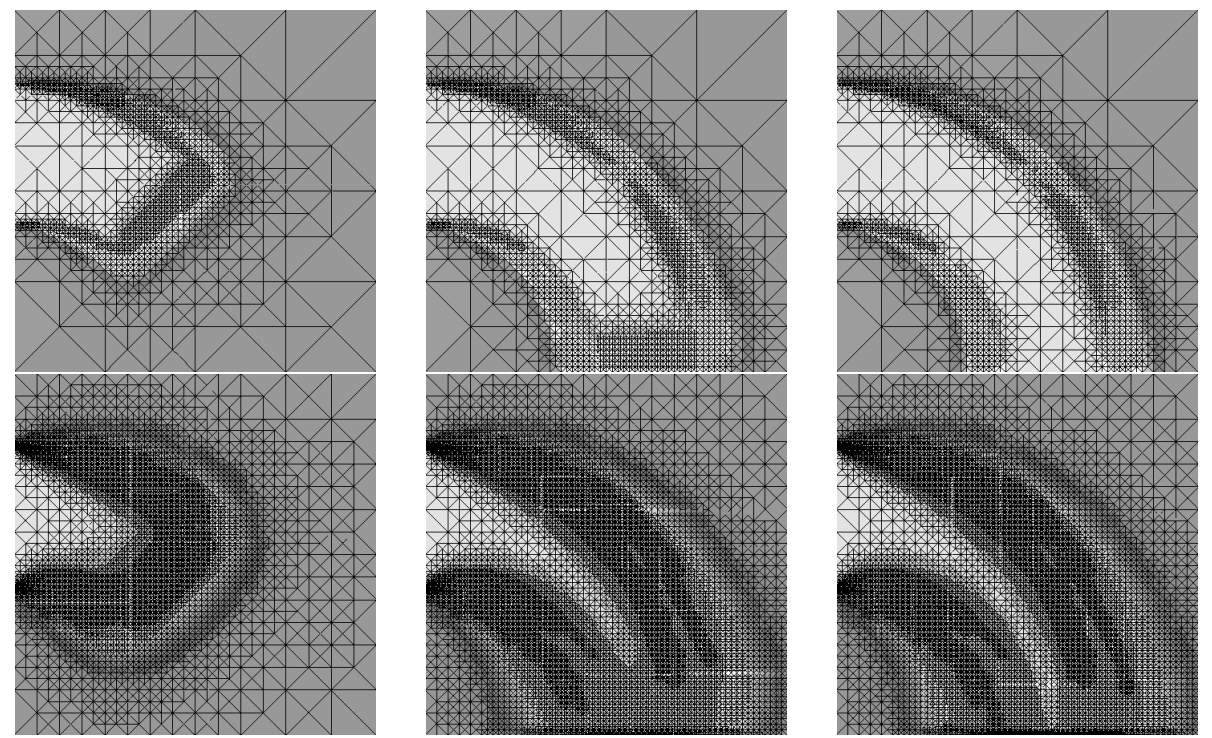

Figure 3. Adaptive solution of the linear problem at $t=\pi / 4, t=$ $\pi / 2$, and $t=2$. A color shading of the solution together with the adaptive grid is plotted for the full upwind flux (top), and the Lax-Friedrichs flux (bottom).

blows up like $h^{-1 / 2}$ which leads to the convergence rate $h^{1 / 6}$ instead of $h^{1 / 3}$. In this sense, the numerical experiments coincide with our theoretical a priori error bound of the error estimator $\eta$. As the $L^{1}$-error itself converges with a rate $h^{1 / 2}$, it is obvious that our error analysis does not give the optimal rate. Nevertheless,

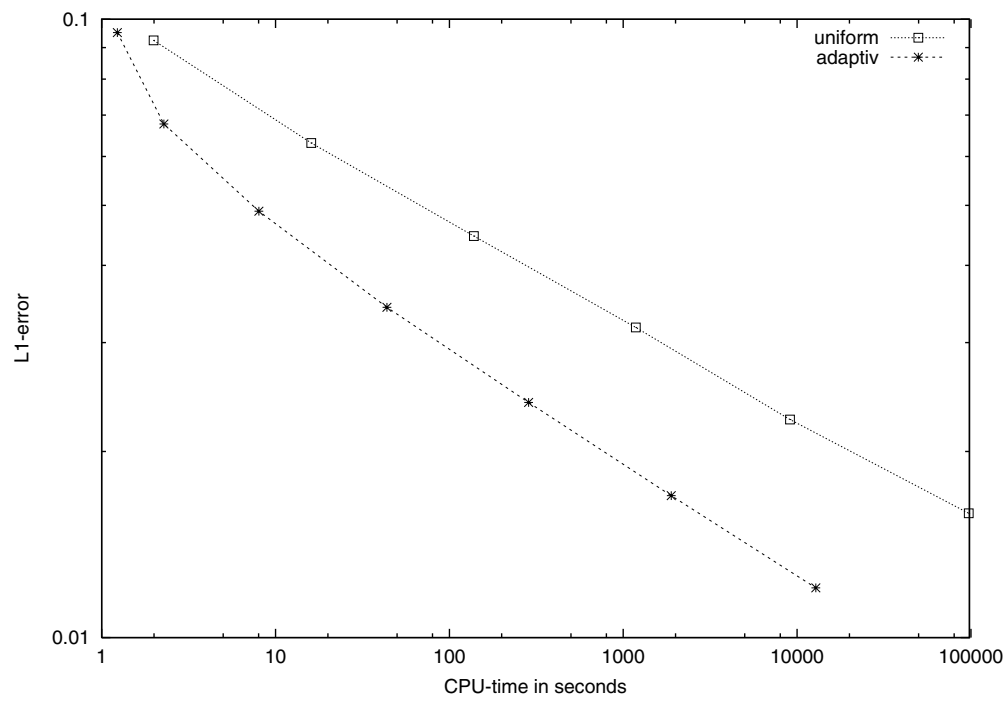

FiguRE 4. $L^{1}$ error versus run time for uniform and adaptive computations for the linear problem. 
we stress that there was no proof of any convergence rate in the general case of bounded domains before.

In a last experiment we analyze the performance of the adaptive scheme versus the same scheme on a mesh with uniform mesh size. Therefore, in Figure 4 we plot the $L^{1}$-error versus run time for uniform and adaptive computations using the upwind flux. The comparison shows that the adaptive scheme performs much better than the method on uniform grids. In addition, we stress that the adaptive algorithm requires far less storage than the uniform one. For instance, in the finest computations the maximal number of mesh cells in the adaptive case was about 350.000, while 4.200.000 mesh cells were used in the uniform computation.

6.3. Second example: Burgers' problem. Next we consider an essential one dimensional problem that we formulate and solve in a two dimensional framework. We look at the following initial boundary value problem in $\Omega:=(0,2) \times(0,1)$ :

$$
\begin{aligned}
c_{t}+\nabla \cdot\left(1 / 2 c^{2}, 0\right)^{\top} & =0 \text { in } \Omega \times(0, T), \\
c(\cdot, 0) & =0 \text { in } \Omega \\
c(x, t) & =\bar{c}(t, x) \text { in } \partial \Omega \times(0, T) .
\end{aligned}
$$

We choose $T=2.0$, and $\bar{c}(t, x)$ is given as

$$
\bar{c}(t, x)=\left\{\begin{aligned}
1 & \text { if } t \in(0,0.5) \cup(1,1.5) \\
-1 & \text { else. }
\end{aligned}\right.
$$

For this problem, the exact solution is given as follows:

If $t \in(0,0.5)$,

$$
c(t, x):= \begin{cases}1, & \text { if } 0 \leq x_{1} \leq 0.5 t \\ 0, & \text { else }\end{cases}
$$

If $t \in(0.5,1.0)$,

$$
c(t, x):= \begin{cases}\frac{x_{1}}{t-0.5}, & \text { if } 0 \leq x_{1}<t-0.5 \\ 1, & \text { if } t-0.5 \leq x_{1}<0.5 t \\ 0, & \text { if } 0.5 t \leq x_{1}<2.0-0.5(t-0.5) \\ -1, & \text { else. }\end{cases}
$$

If $t \in(1.0,1.5)$,

$$
c(t, x):= \begin{cases}1, & \text { if } 0 \leq x_{1}<(t-0.5)-\sqrt{0.5(t-0.5)} \\ \frac{x_{1}}{t-0.5}, & \text { if }(t-0.5)-\sqrt{0.5(t-0.5)} \leq x_{1}<\sqrt{t-0.5} \\ 0, & \text { if } \sqrt{t-0.5} \leq x_{1}<2-0.5(t-0.5) \\ -1, & \text { if } 2-0.5(t-0.5) \leq x_{1}<3-t, \\ -\frac{2-x}{t-1}, & \text { else. }\end{cases}
$$

If $t \in(1.5,2.0)$,

$$
c(t, x):= \begin{cases}\frac{x_{1}}{t-1.5}, & \text { if } 0 \leq x_{1}<t-1.5 \\ 1, & \text { if } t-1.5 \leq x_{1}<t-0.5-\sqrt{0.5(t-0.5)} \\ \frac{x_{1}}{t-0.5}, & \text { if } t-0.5-\sqrt{0.5(t-0.5)} \leq x_{1}<\sqrt{0.5(t-0.5)} \\ 0, & \text { if } \sqrt{0.5(t-0.5)} \leq x_{1}<2-\sqrt{0.5(t-1)} \\ -\frac{2-x_{1}}{t-1}, & \text { if } 2-\sqrt{0.5(t-1)} \leq x_{1}<3-t+\sqrt{0.5(t-1)}, \\ -1, & \text { else. }\end{cases}
$$



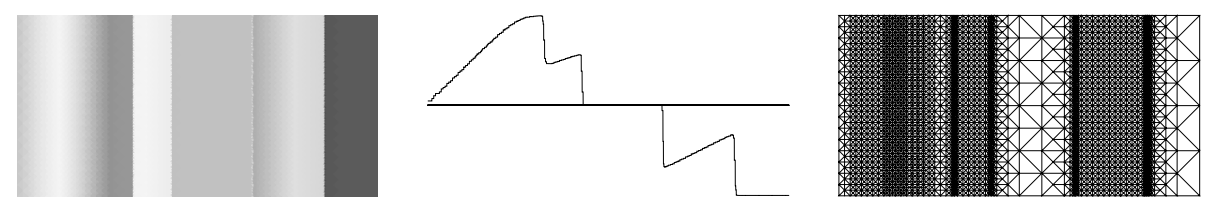

Figure 5. Adaptive solution of the Burgers' problem at $t=2.0$. Color shading of the solution (left), profile on a horizontal cut through the domain (middle) and adaptive grid (right).

We chose this test problem in order to demonstrate the behavior of the error estimator and the resulting numerical scheme in the case where the inflow and outflow boundaries change in time, dependent on the boundary values and the solution. Figure 5 shows the adaptive numerical solution and computational grid for $t=2.0$ for a moderately prescribed error tolerance. We see that the grid is refined in the shock regions and also moderately in the regions of the rarefaction waves, while a very coarse resolution is needed in the regions of constant states.

Figure 6 demonstrates the adaptive refinement around $t=1$. For $t$ slightly smaller than one, no heavy refinement is needed near the boundaries which is automatically reflected by the error estimator. At $t=1$ the boundary values change, and immediately the boundary zones are heavily refined. This example shows that the adaptive algorithm is capable to detect the sources of errors coming from the approximation of the boundary values automatically.

Finally, we compare the adaptive and uniform algorithms in an error versus run time plot (see Figure 17). Although the algorithm on uniform grids converges with a rate of $h$ in the case of the Burgers' problem, and the error estimator $\eta$ still converges like $h^{1 / 3}$, the adaptive algorithm performs better than the uniform one, and requires far less storage.
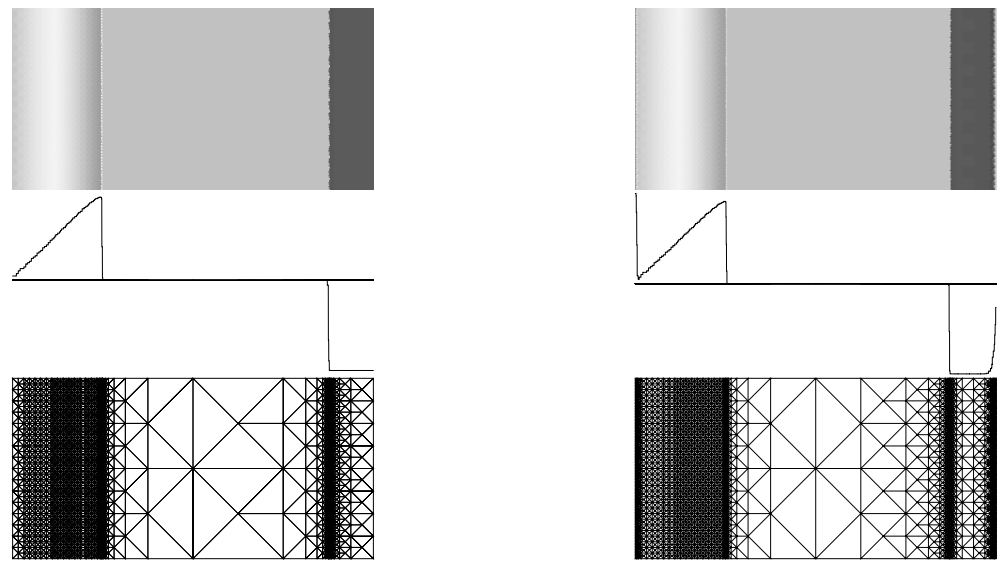

Figure 6. Adaptive solution of the Burgers' problem at $t=0.997$ (left), and $t=1.016$ (right). Color shading of the solution (top), profile on a horizontal cut through the domain (middle) and adaptive grid (bottom). 


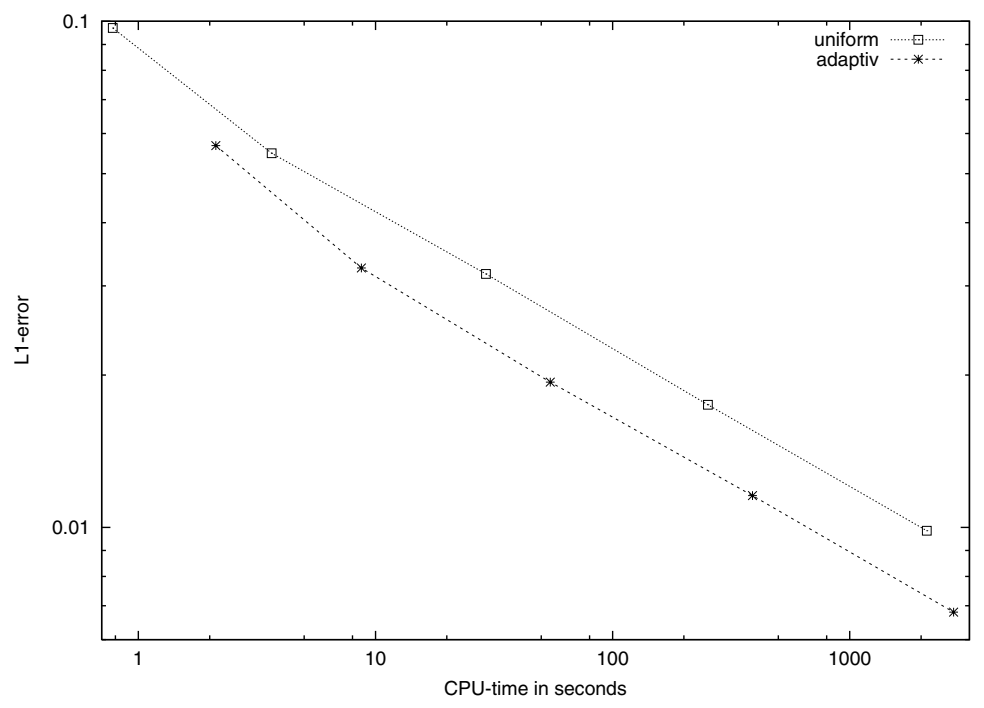

Figure 7. $L^{1}$ error versus run time for the Burgers' problem.

\section{Appendix A. $B V$ estimate of the ExaCt solution on CONVEX Domains}

Under certain regularity hypotheses on the data and on the boundary of $\Omega$, the $B V$ estimate (77) has been proved by Bardos, LeRoux and Nédélec [BLN79] (see also [MNRR96]). They obtain the estimate from a uniform $B V$ estimate on viscous approximations $u^{\varepsilon}$ of problem (11)-(3), $\varepsilon$ being the viscosity parameter. In our considerations $\Omega$ is only a polygonal open bounded set, which makes it difficult to adapt the technique developed in BLN79] (the solution $u^{\varepsilon}$ of the viscous approximation of problem (11)-(3) is not regular enough in that case). In order to prove the $B V$ estimate (7) in our situation, we will follow the approach of ChainaisHillairet [CH99a for unbounded domains. Thus, we construct a sequence of finite volume approximations to (11)-(3) on structured meshes and drive a uniform bound on the $B V$ semi-norm. Technical difficulties arise from the fact that we consider a cartesian grid on a polygonal open set which is possibly not rectangular. For simplicity of our presentation we will suppose that the flux function $\mathbf{F}$ is constant with respect to $(x, t)$, i.e., $\mathbf{F}(x, t, u)=\mathbf{F}(u)$.

Notation for a structured mesh approximation of $\Omega$. Let $\left(\mathbf{e}_{i}\right)_{1, d}$ be an orthonormal basis of $\mathbb{R}^{d}$, and $O \in \mathbb{R}^{d}$ a fixed origin. Then $\left(O, \mathbf{e}_{1}, \ldots, \mathbf{e}_{d}\right)$ is an affine basis of $\mathbb{R}^{d}$ viewed as an affine space. Given $h>0$, we define the structured mesh $\mathcal{T}_{h}\left(\mathbb{R}^{d}\right)$ as

$$
\mathcal{T}_{h}\left(\mathbb{R}^{d}\right):=\left\{T_{j} \mid T_{j}:=\left(j_{1} h,\left(j_{1}+1\right) h\right) \times \cdots \times\left(j_{d} h,\left(j_{d}+1\right) h\right), j \in \mathbb{Z}^{d}\right\}
$$

and set

$$
\mathcal{T}_{h}:=\mathcal{T}_{h}(\Omega):=\left\{T \in \mathcal{T}_{h}\left(\mathbb{R}^{d}\right) \mid \bar{T} \subset \Omega\right\}, \quad \bar{\Omega}_{h}=\bigcup_{T \in \mathcal{T}_{h}} \bar{T} .
$$

Define $\Omega_{h}$ as the interior of $\bar{\Omega}_{h}$. Furthermore, the time step $\Delta t$ is defined as $\Delta t:=\frac{T}{N}$, where $N=N(h)$ is given, and we denote $J:=\left\{t_{0}, t_{1}, \ldots, t_{N}\right\}$ where $t_{n}:=n \Delta t$. Using multi-index notation, we define the set of neighboring cells to 
a cell $T_{j}$ by $\mathcal{N}(j):=\left\{T_{l} \in \mathcal{T}_{h}\left(\mathbb{R}^{d}\right) \mid l=j \pm \mathbf{e}_{i}, i=1, \ldots, d\right\}$ and denote the face between two neighboring cells by $S_{j, l}:=\overline{T_{j}} \cap \overline{T_{l}}$. Furthermore, set

$$
\mathcal{E}:=\left\{S_{j, l} \neq \emptyset \mid T_{j}, T_{l} \in \mathcal{T}_{h}\right\}, \quad \partial \mathcal{E}:=\left\{S_{j, l} \neq \emptyset \mid T_{j} \in \mathcal{T}_{h}, T_{l} \in \mathcal{T}_{h}\left(\mathbb{R}^{d}\right) \backslash \mathcal{T}_{h}\right\} .
$$

In the next step we introduce some notation that allows us to link the exterior faces $S \in \partial \mathcal{E}$ to its orthogonal projection $P(S)$ on the closest part of $\partial \Omega$. Therefore, let us denote $A_{1}, \ldots, A_{n}$ the distinct faces of $\Omega$ which generate the hyperplanes $H_{1}, \ldots, H_{n}$. For $S \in \partial \mathcal{E}$ define $\mathcal{I}_{S}=\left\{i \in\{1, \ldots, n\} \mid \operatorname{dist}\left(S, A_{i}\right) \leq 2 D_{d} h\right\}$, where $D_{d}:=\sqrt{2^{d-1}}$ is the diameter of the unit cube in $\mathbb{R}^{d}$. Let $P_{i}$ denote the orthogonal projection on $H_{i}$, and define $G_{i}$ as the cylinder generated by $A_{i}$ : $G_{i}=P_{i}^{-1}\left(A_{i}\right)$. We define the set of faces associated with the boundary part $A_{i}$ as

$$
\partial \mathcal{E}_{i}:=\left\{S \in \partial \mathcal{E} \mid \mathcal{I}_{S}=\{i\} \text { and } S \subset G_{i}\right\},
$$

and the remaining boundary faces as

$$
\partial \mathcal{E}_{\bullet}:=\partial \mathcal{E} \backslash \bigcup_{i=1}^{n} \partial \mathcal{E}_{i}
$$

Finite volume approximation on $\mathcal{T}_{h} \times J$. For the finite volume approximation on $\mathcal{T}_{h} \times J$, we first define the discrete initial and boundary values as

$$
\begin{aligned}
c_{j}^{0} & :=\frac{1}{\left|T_{j}\right|} \int_{T_{j}} c_{0} \quad \forall T_{j} \in \mathcal{T}_{h}, \\
\bar{c}_{j l}^{n} & :=\frac{1}{\Delta t\left|P_{i}\left(S_{j l}\right)\right|} \int_{t^{n}}^{t^{n+1}} \int_{P_{i}\left(S_{j l}\right)} \bar{c}(x, t) d \gamma(x) \mathrm{dt} \quad \forall S_{j l} \in \partial \mathcal{E}_{i}, \\
\bar{c}_{j l}^{n} & :=0 \quad \forall S_{j l} \in \partial \mathcal{E}_{\bullet} .
\end{aligned}
$$

Then for all $t^{n+1} \in J$ and all $T_{j} \in \mathcal{T}_{h}$, the values $c_{j}^{n+1}$ are defined as

$$
c_{j}^{n+1}:=c_{j}^{n}-\frac{\Delta t}{h} \sum_{l \in N(j)}\left(F_{j l}\left(c_{j}^{n}\right)-F_{j l}\left(c_{j l}^{n}\right)\right), \quad c_{j l}^{n}:= \begin{cases}c_{l}^{n}, & \text { if } T_{l} \in \mathcal{T}_{h}, \\ \bar{c}_{j l}^{n}, & \text { else },\end{cases}
$$

where we use a flux splitting approach with monotone fluxes

$$
F_{j l}(c):=\frac{1}{2}\left(-\mathbf{F}(c) \cdot \mathbf{n}_{j l}+\mathcal{L} c\right),
$$

where $\mathcal{L}$ denotes the Lipschitz constant of $\mathbf{F}$. The numerical approximation $c_{h}$ of the entropy solution $c$ is given by

$$
c_{h}(x, t)= \begin{cases}c_{j}^{n} & \text { if }(x, t) \in T_{j} \times\left[t^{n}, t^{n+1}\right), T_{j} \in \mathcal{T}_{h}, \\ 0 & \text { otherwise. }\end{cases}
$$

Although the value given in (51) is arbitrary, the error in the implementation of the finite volume method induced by such a process does not affect the convergence of the scheme because the number of interfaces $S$ "close" to a corner of $\partial \Omega$ is at most of order $h /|S|$ where $|S|=h^{d-1}$ is the Lebesgue measure of $S$. We specify this point in the following lemma.

Lemma A.1. For sufficiently small $h$, the set $\partial \mathcal{E} \bullet$ is finite and there exists a constant $C_{\#}$ which depends on the dimension d and on $\Omega$ only such that the cardinal of $\partial \mathcal{E} \bullet$ is bounded by $\frac{C_{\#}}{h^{d-2}}$. 
Proof. For $X \subset \mathbb{R}^{d}$ and $a>0$, we define the $a$ neighborhood of $X$ as $\mathcal{V}_{a}(X):=$ $\left\{y \in \mathbb{R}^{d}|\operatorname{dist}(y, X)|<a\right\}$. Let $H$ and $G$ be two affine hyperplanes of $\mathbb{R}^{d}$, and denote by $\overline{H G}$ the angle between them. We will show in a first step that for $H, G$ nonparallel, i.e., $\overline{H G} \neq 0$, it holds that

$$
\mathcal{V}_{a}(H) \cap \mathcal{V}_{a}(G) \subset \mathcal{V}_{\left(\frac{3 \pi}{2 H G}+1\right) a}(H \cap G) .
$$

Consider a point $U \in \mathcal{V}_{a}(H) \cap \mathcal{V}_{a}(G)$. Denote by $U_{H \cap G}, U_{H}$ and $U_{G}$ the orthogonal projections of $U$ on $H \cap G, H$ and $G$, respectively, and by $U_{H G}$ the orthogonal projection of $U_{H}$ on $G$. We have

$$
\begin{aligned}
\operatorname{dist}(U, H \cap G) & =\left|U_{H \cap G}-U\right| \\
& \leq\left|U_{H \cap G}-U_{H}\right|+\left|U_{H}-U\right|=\frac{\left|U_{H}-U_{H G}\right|}{\sin (\overline{H G})}+\left|U_{H}-U\right| \\
& \leq \frac{\left|U_{H}-U\right|+\left|U-U_{G}\right|+\left|U_{G}-U_{H G}\right|}{\sin (\overline{H G})}+\left|U_{H}-U\right|,
\end{aligned}
$$

and we conclude (54) by using the estimates

$$
\left|U_{G}-U_{H G}\right| \leq\left|U-U_{H}\right|<a, \quad\left|U-U_{G}\right|<a,
$$

and

$$
\frac{1}{\sin (\overline{H G})} \leq \frac{\pi}{2 \overline{H G}}
$$

For

$$
h<h_{0}:=\frac{1}{8 D_{d}} \min _{1 \leq i, j \leq n}\left\{\operatorname{dist}\left(A_{i}, A_{j}\right), A_{i} \cap A_{j}=\emptyset\right\},
$$

the intersection $\mathcal{V}_{4 D_{d} h}\left(A_{i}\right) \cap \mathcal{V}_{4 D_{d} h}\left(A_{j}\right)$ is not empty if and only if $A_{i}$ and $A_{j}$ are adjacent. Furthermore, if $A_{i} \neq A_{j}$, then $\overline{A_{i} A_{j}}>0$, and (54) yields

$$
\mathcal{V}_{4 D_{d} h}\left(A_{i}\right) \cap \mathcal{V}_{4 D_{d} h}\left(A_{j}\right) \subset \mathcal{V}_{C_{1} h}\left(A_{i} \cap A_{j}\right)
$$

to where $C_{1}:=\max _{1 \leq k \neq l \leq n}\left\{\left(\frac{3 \pi}{2 \overline{A_{k} A_{l}}}+1\right) 4 D_{d} h, A_{k} \cap A_{l} \neq \emptyset\right\}$.

Let $S \in \partial \mathcal{E}_{\bullet}$. We will show that $S \subset \mathcal{V}_{C_{1} h}\left(A_{i} \cap A_{j}\right)$ for some adjacent $A_{i}, A_{j}$. This assertion is clearly satisfied if $\{i, j\} \subset \mathcal{I}_{S}$. If there is no such subset $\{i, j\}$, there is an $i \in \mathcal{I}_{S}$ with $S \nsubseteq G_{i}$. In that case $\operatorname{dist}\left(S, \partial A_{i}\right)=\operatorname{dist}\left(S, A_{i}\right) \leq 2 D_{d} h$, and hence there exists $x \in S, y \in \partial A_{i}$ such that $|x-y| \leq 2 D_{d} h$. Let $j \neq i$ be such that $y \in A_{i} \cap A_{j}$. We have $x \in \mathcal{V}_{2 D_{d} h}\left(A_{i}\right) \cap \mathcal{V}_{2 D_{d} h}\left(A_{j}\right)$, and, since $\operatorname{diam}(S)=D_{d} h$, $S \subset \mathcal{V}_{4 D_{d} h}\left(A_{i}\right) \cap \mathcal{V}_{4 D_{d} h}\left(A_{j}\right) \subset \mathcal{V}_{C_{1} h}\left(A_{i} \cap A_{j}\right)$.

As a consequence, every $S \in \partial \mathcal{E}_{\bullet}$ is subset of a disk $\Delta^{2}$ of radius $C_{1} h$ if $d=2$, of a cylinder $\Delta^{3}$ of radius $C_{1} h$ if $d=3$. The cardinal of $\Delta^{d} \cap \mathcal{T}$ is of order $h^{-(d-2)}$ and every cell of $\mathcal{T}$ has $2 d$ edges. This proves $\# \partial \mathcal{E} \bullet \leq \frac{C_{\#}}{h^{d-2}}$.

In the next lemma we are going to prove some basic properties of the projection $P_{i}(S)$ for $S \in \partial \mathcal{E}_{i}$.

Lemma A.2. (1) There exists $C_{0}>0$ which depends on $d$ and $\Omega$ only such that

$$
\forall i \in\{1, \ldots, n\} \forall S \in \partial \mathcal{E}_{i}, \quad|S| \leq C_{0}\left|P_{i}(S)\right| .
$$

(2) If $S, S^{\prime} \in \partial \mathcal{E}_{i}$, then $P_{i}(S) \cap P_{i}\left(S^{\prime}\right)=\emptyset$ if, and only if, $S \cap S^{\prime}=\emptyset$. 
Proof. (1) Let $H$ be an affine hyperplane of $\mathbb{R}^{d}$, and let $P$ denote the orthogonal projection on $H$. It is then elementary to show that

$$
|P(S)|=|S| \cos (\overline{H S})
$$

for all $S \in \mathcal{E}$, where $\overline{H S}$ denotes the angle between $H$ and the hyperplane $\langle S\rangle$ generated by $S$.

Let $A_{i}$ be one of the polygonal boundary faces of $\Omega$. First suppose that $A_{i}$ is parallel to one of the canonical hyperplanes generated by the basis $\left(\mathbf{e}_{i}\right)_{1, d}$. Then any elements of $\mathcal{E}$ is either orthogonal or parallel to $H_{i}$. Let $S_{j l} \in \partial \mathcal{E}_{i}$ and suppose that $S_{j l}$ is orthogonal to $H_{i}$ (in which case $\left|P_{i}(S)\right|=0$ by (566)). As $S_{j l} \in \partial \mathcal{E}_{i}$, we have $\overline{T_{l}} \cap \partial \Omega \neq \emptyset$ : there exists a $A_{k}$ such that $\overline{T_{l}} \cap A_{k} \neq \emptyset$ and, since $\max _{x \in S_{j l}, y \in \overline{T_{l}}}|x-y|=$ $D_{d}$, we have $k \in \mathcal{I}_{S}$. Furthermore, $k \neq i$ (for $T_{l}$ is a subset of the cylinder generated by $S$ and the normal $\mathbf{n}_{S}$ to the hyperplane $\langle S\rangle$, a cylinder which is parallel to $A_{i}$ ), and this contradicts the fact that $S_{j l} \in \partial \mathcal{E}_{i}$. Consequently $S_{j l}$ is parallel to $H_{i}$ and $\left|P\left(S_{j l}\right)\right|=\left|S_{j l}\right|$.

Suppose now that $H_{i}$ is not parallel to any of the hyperplanes generated by $\left(\mathbf{e}_{i}\right)_{1, d}$, and define

$$
\alpha_{i}:=\max \left\{\overline{H_{i}\left\langle\left(\mathbf{e}_{k}\right)_{k \in J}\right\rangle} ; J \subset\{1, \ldots, d\}, \# J=d-1\right\}<\frac{\pi}{2} .
$$

Then, for every $S \in \mathcal{E}$, we have, by (56),

$$
\left|P_{i}(S)\right| \geq|S| \cos \left(\alpha_{i}\right) .
$$

Consequently, we have shown that (155) holds with $C_{0}^{-1}:=\min _{1 \leq i \leq n} \cos \left(\alpha_{i}\right)>0$.

(2) Let us prove the second part of Lemma A.2 Let $S_{j l}, S_{n m} \in \partial \mathcal{E}_{i}$. Obviously, $P_{i}\left(S_{j l}\right) \cap P_{i}\left(S_{n m}\right)=\emptyset \Rightarrow S_{j l} \cap S_{n m}=\emptyset$. We suppose that $P_{i}\left(S_{j l}\right) \cap P_{i}\left(S_{n m}\right) \neq \emptyset$ and intend to prove that $S_{j l} \cap S_{n m} \neq \emptyset$. Let $x_{i} \in P_{i}\left(S_{j l}\right) \cap P_{i}\left(S_{n m}\right)$, and let $D_{i}$ be the line $P_{i}^{-1}\left(\left\{x_{i}\right\}\right)$. Let $z \in D_{i} \cap S_{j l}, z^{\prime} \in D_{i} \cap S_{n m}$. If $x_{i}=z=z^{\prime}$, we are done. Thus, suppose $x_{i} \neq z$ and set $\mathbf{u}:=\frac{z-x_{i}}{\left|z-x_{i}\right|}$. Then $t \mapsto x_{i}+t \mathbf{u}$ is a parametrization of $D_{i}$. We have $z=x_{i}+t_{z} \mathbf{u}$ with $t_{z}=\left|x_{i}-z\right|>0$, and $z^{\prime}=x_{i}+t_{z}^{\prime} \mathbf{u}$ with $t_{z}^{\prime} \in \mathbb{R}$. In fact, $t_{z}^{\prime}>0$; otherwise, we would have $x_{i} \in\left[z, z^{\prime}\right] \subset \Omega$ (by convexity of $\Omega$ ) and this contradicts $x_{i} \in A_{i} \subset \partial \Omega$. Suppose that $S_{j l} \cap S_{n m}=\emptyset$. Then $t_{z}^{\prime} \neq t_{z}$ and, for example, $t_{z}^{\prime}>t_{z}$. We will show that $T_{m} \in \mathcal{T}_{h}$, which contradicts $S_{n m} \in \partial \mathcal{E}_{i}$. Denote by $H_{i}^{+}$the open affine half-space generated by $H_{i}$ and $\mathbf{u}$. Let $J_{0}=\left\{k \in\{1, \ldots, d\}, \mathbf{u} \cdot \mathbf{e}_{k} \neq 0\right\}$ and $I_{0}=\{1, \ldots, d\} \backslash J_{0}$. Define $\left(\varepsilon_{k}\right)_{1, d}$ by $\varepsilon_{k}=\operatorname{sgn}\left(\mathbf{u} \cdot \mathbf{e}_{k}\right)$ if $k \in J_{0}, \varepsilon_{k} \in\{-1,1\}$ if $k \in I_{0}$, and set $Q_{\mathbf{u}} i=\prod_{k=1}^{d} \mathrm{I}\left(0, \varepsilon_{k}\right)$, where $\mathrm{I}\left(0, \varepsilon_{k}\right)$ denotes the interval $(0,1)$ if $\varepsilon_{k}=1,(-1,0)$ if $\varepsilon_{k}=-1$. For each $T_{p}=p+(0, h)^{d} \in \mathcal{T}_{h}\left(\mathbb{R}^{d}\right)$, there exists a unique $p_{\mathbf{u}} \in h \mathbb{Z}^{d}$ such that $T_{p}=p_{\mathbf{u}}+h Q_{\mathbf{u}}$ (actually, $p_{\mathbf{u}}$ reads $\left.p_{\mathbf{u}}=O+\sum_{k=1}^{d}\left(p_{k}+\left(\varepsilon_{k}\right)^{-}\right) \mathbf{e}_{k}\right)$.

These notations being set, we prove that for $T_{p}$ with $\overline{T_{p}} \cap\{z+t \mathbf{u}, t>0\} \neq \emptyset$ we have $\overline{T_{p}} \subset H_{i}^{+}$. We work in the affine base $\left(j_{\mathbf{u}}, \varepsilon_{1} \mathbf{e}_{1}, \ldots, \varepsilon_{d} \mathbf{e}_{d}\right)$ (recall that $j_{\mathbf{u}}$ is such that $\left.T_{j}=j_{\mathbf{u}}+Q_{\mathbf{u}}\right)$, and denote by $\left(\widetilde{x}_{k}\right)_{1, d}$ the coordinate of a point $x \in \mathbb{R}^{d}$. Let $y \in \overline{T_{p}} \cap\{z+t \mathbf{u}, t>0\}$, set $Z_{y}=\left\{k \in\{1, \ldots, d\}, \widetilde{y}_{k} \in h \mathbb{Z}\right\}$, and $T_{y}=\left\{x \in \mathbb{R}^{d}, 0<\widetilde{x}_{k}-h \beta_{k}<h\right\}$, where $\beta_{k}=\left[\widetilde{y}_{k} / h\right]$ is the unique integer such that $\beta_{k} \leq\left[\widetilde{y}_{k} / h\right]<\beta_{k}+1$. Since $y \in \overline{T_{p}}$, we have $T_{p}=T_{y}$ or $T_{p}=T_{y}-\mathbf{e}_{k}$ for a $k \in Z_{y}$. Besides, $y=z+t \mathbf{u}$ for a positive $t$ and $\widetilde{y}_{k}=\left(y-j_{\mathbf{u}}\right) \cdot \varepsilon_{k} \mathbf{e}_{k}$; therefore, $\widetilde{y}_{k}=\left(z-j_{\mathbf{u}}\right) \cdot \varepsilon_{k} \mathbf{e}_{k}+t \varepsilon_{k} \mathbf{u} \cdot \mathbf{e}_{k}$. In the new coordinates, we have $\overline{T_{j}}=\left\{x \in \mathbb{R}^{d}\right.$, $\left.0 \leq \widetilde{x}_{k} \leq h\right\}$ and since $z \in \overline{T_{j}}$, the term $\left(z-j_{\mathbf{u}}\right) \cdot \varepsilon_{k} \mathbf{e}_{k}$ is nonnegative. By definition of $\varepsilon_{k}$, the term $t \varepsilon_{k} \mathbf{u} \cdot \mathbf{e}_{k}$ is also nonnegative, and, if $k \in J_{0}$, it is positive. In 
particular, if $k \in Z_{y} \cap J_{0}$, then $\widetilde{y}_{k} \geq h$ and $\beta_{k} \geq 1$. This implies that $\overline{T_{p}} \subset C_{\mathbf{u}}:=$ $\left\{x \in \mathbb{R}^{d}, \forall k \in J_{0}, \widetilde{x}_{k} \geq 0\right\}$. If $x \in \mathbb{R}^{d}$, we have $\left(x-j_{\mathbf{u}}\right) \cdot \mathbf{u} i=\sum_{k=1}^{d} \widetilde{x}_{k} \varepsilon_{k} \mathbf{e}_{k}$, therefore, $C_{\mathbf{u}} \subset\left\{x \in \mathbb{R}^{d},\left(x-j_{u}\right) \cdot \mathbf{u} \geq 0\right\}$, and this last set is itself a subset of $H_{i}^{+}$ for $j_{\mathbf{u}} \in H_{i}^{+}$(we have $T_{j} \subset H_{i}^{+}$). This proves $\overline{T_{p}} \subset H_{i}^{+}$.

Since $z^{\prime} \in \overline{T_{m}} \cap\{z+t \mathbf{u}, t>0\}$, this last intersection is not empty and $\overline{T_{m}} \subset H_{i}^{+}$. This entails $\overline{T_{m}} \subset \Omega$, which contradicts the hypothesis that $T_{m} \in \mathcal{T}_{h}\left(\mathbb{R}^{d}\right) \backslash \mathcal{T}_{h}(\Omega)$. Therefore, we have $S_{j l} \cap S_{n m} \neq \emptyset$.

Proof of a uniform bound on the $B V$ norm of $c_{h}$. Now we have two tasks. First we show that $c_{h}$ is indeed a numerical approximation of $c$ (Lemma A.3), and second we establish a uniform bound on the $B V$ norm of $c_{h}$ (Lemma A.4).

Lemma A.3. Let $c_{h}$ be defined by (53). Assume that Assumption 3.1 holds. Then, under the CFL condition

$$
\Delta t \leq \frac{(1-\xi)}{2(d-1) \mathcal{L}} h, \quad \xi \in(0,1)
$$

the sequence $\left(c_{h}\right)$ converges to the entropy solution $c$ of the problem (11)-(13) in $L^{1}(\Omega \times(0, T))$.

Sketch of the proof. Recall that, by the maximum principle, the sequence $\left(c_{h}\right)$ is uniformly bounded with respect to $h$ in $L^{\infty}(\Omega \times(0, T))$. We first make reference to Vov02 where the following result is proved. Given a nonnegative function $\varphi \in C_{c}^{\infty}\left(\mathbb{R}^{d} \times \mathbb{R}^{+}\right)$, there exists $\varepsilon_{h}:=\varepsilon_{h}(\varphi)$ such that $\lim _{h \rightarrow 0} \varepsilon_{h}=0$ and

$$
\begin{aligned}
\int_{0}^{T} \int_{\Omega_{h}}\left(c_{h}-\kappa\right)^{ \pm} \partial_{t} \varphi & +\Phi^{ \pm}\left(c_{h}, \kappa\right) \cdot \nabla \varphi+\int_{\Omega_{h}}\left(c_{h}^{0}-\kappa\right)^{ \pm} \varphi(\cdot, 0) \\
& +\mathcal{L} \sum_{n=0}^{N} \sum_{S_{j l} \in \partial \mathcal{E}} \Delta t\left|S_{j l}\right|\left(\bar{c}_{j l}^{n}-\kappa\right)^{ \pm} \varphi_{j l}^{n} \geq-\varepsilon_{h},
\end{aligned}
$$

where $\varphi_{j l}^{n}:=\frac{1}{\Delta t\left|S_{j l}\right|} \int_{t^{n}}^{t^{n+1}} \int_{S_{j l}} \varphi(x, t) \mathrm{dx} \mathrm{dt}$.

We intend to prove the following bound for a constant $C>0$, and $\tilde{\varepsilon}_{h}:=\tilde{\varepsilon}_{h}(\varphi)$ such that $\lim _{h \rightarrow 0} \tilde{\varepsilon}_{h}=0$ :

$$
\sum_{n=0}^{N} \sum_{S_{j l} \in \partial \mathcal{E}} \Delta t\left|S_{j l}\right|\left(\bar{c}_{j l}^{n}-\kappa\right)^{ \pm} \varphi_{j l}^{n} \leq C \int_{\partial \Omega \times(0, T)}(\bar{c}-\kappa)^{ \pm} \varphi d \gamma(x) \mathrm{dt}+\tilde{\varepsilon}_{h} .
$$

Indeed, suppose for a moment that (59) holds. We then conclude the proof with the following arguments. The Lebesgue measure $\left|\Omega \backslash \Omega_{h}\right|=\mathcal{O}(h)$, because $\Omega \backslash \Omega_{h} \subset$ $\mathcal{V}_{D_{d} h}(\partial \Omega)$. Consequently, since $\left(c_{h}-\kappa\right)^{ \pm} \partial_{t} \varphi+\Phi^{ \pm}\left(c_{h}, \kappa\right) \cdot \nabla \varphi$ and $\left(c_{h}^{0}-\kappa\right)^{ \pm} \varphi(\cdot, 0)$ are uniformly bounded with respect to $h$ in $L^{\infty}(\Omega \times(0, T))$, and $L^{\infty}(\Omega)$, respectively, we have with (59)

$$
\begin{array}{r}
\int_{0}^{T} \int_{\Omega}\left(c_{h}-\kappa\right)^{ \pm} \partial_{t} \varphi+\Phi^{ \pm}\left(c_{h}, \kappa\right) \cdot \nabla \varphi+\int_{\Omega}\left(c_{0}^{h}-\kappa\right)^{ \pm} \varphi(\cdot, 0) \\
+C \mathcal{L} \int_{\partial \Omega \times(0, T)}(\bar{c}-\kappa)^{ \pm} \varphi d \gamma(x) \mathrm{dt} \geq-\bar{\varepsilon}_{h},
\end{array}
$$

where $\lim _{h \rightarrow 0} \bar{\varepsilon}_{h}=0$. Since $\left(c_{h}^{0}\right)$ is converging to $c_{0}$ in $L^{1}(\Omega)$, the uniform bound on $\left(c_{h}\right)$ and (60) are sufficient to get a subsequence of $\left(c_{h}\right)$ that converges in the nonlinear weak- $\star$ sense to an entropy process solution $\widetilde{c}$ of problem (1)-(3). Let us note 
that the constant factor in front of the boundary term $\int_{\partial \Omega \times(0, T)}(\bar{c}-\kappa)^{ \pm} \varphi d \gamma(x) \mathrm{dt}$ in the definition of the entropy solution is required to be any bound on the Lipschitz semi-norm of $\mathbf{F}$, and not the Lipschitz constant of $\mathbf{F}$. By a uniqueness result, this entropy process solution turns out to be the entropy solution $c$ of problem (1)-(3), and this shows that the whole sequence $\left(c_{h}\right)$ converges to $c$ in $L^{1}(\Omega \times(0, T))$. We refer to Vov02 for details on the terminology and the results.

Let us turn to the proof of (59). Denote by $U_{h}$ the left hand side of (59). We have $U_{h}=\sum_{i=1}^{n} U_{i}+U_{\bullet}$ with

$$
U_{i}=\sum_{n=0}^{N} \sum_{S_{j l} \in \partial \mathcal{E}_{i}} \Delta t\left|S_{j l}\right|\left(\bar{c}_{j l}^{n}-\kappa\right)^{ \pm} \varphi_{j l}^{n}, \quad U_{\bullet}=\sum_{n=0}^{N} \sum_{S_{j l} \in \partial \mathcal{E} \bullet} \Delta t\left|S_{j l}\right|\left(\bar{c}_{j l}^{n}-\kappa\right)^{ \pm} \varphi_{j l}^{n} .
$$

Since $\left(\bar{c}_{j l}^{n}-\kappa\right)^{ \pm} \varphi_{j l}^{n} \leq\left(\left\|\bar{c}_{j l}^{n}\right\|_{L^{\infty}}+|\kappa|\right)\|\varphi\|_{L^{\infty}}$, we have, by Lemma A.1, $0 \leq U_{\bullet} \leq$ $C h$ for a constant $C$ independent of $h$, and $\lim _{h \rightarrow 0} \bar{U}_{\bullet}=0$. For $i \in\{1, \ldots, n\}$, and $S_{j l} \in \partial \mathcal{E}_{i}$, where $\bar{c}_{j l}^{n}$ is defined as the mean value of $\bar{c}$ over $\left(A_{i} \cap P_{i}\left(S_{j l}\right)\right) \times\left(t^{n}, t^{n+1}\right)$. Since $s \mapsto(s-\kappa)^{ \pm}$is convex, we have by Jensen's inequality

$$
\left(\bar{c}_{j l}^{n}-\kappa\right)^{ \pm} \leq \frac{1}{\Delta t\left|A_{i} \cap P_{i}\left(S_{j l}\right)\right|} \int_{t^{n}}^{t^{n+1}} \int_{A_{i} \cap P_{i}\left(S_{j l}\right)}(\bar{c}(x, t)-\kappa)^{ \pm} d \gamma(x) \mathrm{dt} .
$$

Taking (55) into account, we get

$$
\bar{U}_{i} \leq C_{1} \sum_{n=0}^{N} \sum_{S_{j l} \in \partial \mathcal{E}_{i}} \int_{t^{n}}^{t^{n+1}} \int_{A_{i} \cap P_{i}\left(S_{j l}\right)}(\bar{c}(x, t)-\kappa)^{ \pm} d \gamma(x) \mathrm{dt} \varphi_{j l}^{n} .
$$

By Lemma A.2(2) and the regularity of the function $\varphi$ we have

$$
U_{i} \leq C \int_{0}^{T} \int_{A_{i}}(\bar{c}(x, t)-\kappa)^{ \pm} \varphi d \gamma(x) \mathrm{dt}+\tilde{\varepsilon}_{h}^{i},
$$

where $\tilde{\varepsilon}_{h}^{i} \rightarrow 0$ when $h \rightarrow 0$. Hence, (59) follows with $\tilde{\varepsilon}_{h}=\sum_{i=1}^{n} \tilde{\varepsilon}_{h}^{i}+\bar{U} \bullet$.

Lemma A.4. Under the assumptions of Lemma A.3, there exists a constant $C_{B V}>$ 0 , which depends on the data and on $\Omega$ only such that

$$
\left\|c_{h}\right\|_{B V\left(\Omega_{h} \times(0, T)\right)} \leq C_{B V} .
$$

Proof. For $B V$ data, the bounded character of the variation with respect to time of the solution of an explicit finite volume scheme with monotone fluxes is ensured independently of the structure of the mesh (see CCL95b for example). On the contrary, the bounded character of the variation in space of the same numerical solution remains an open question in the case where an unstructured mesh is used. This is the reason why we used a cartesian grid to defined $c_{h}$. We decompose $\left\|c_{h}\right\|_{B V\left(\Omega_{h} \times(0, T)\right)}=B V_{t}+B V_{x}$ with

$$
B V_{t}:=\sum_{n=0}^{N} \sum_{T_{j} \in \mathcal{T}_{h}}\left|T_{j}\right|\left|c_{j}^{n+1}-c_{j}^{n}\right|, \quad B V_{x}:=\sum_{n=0}^{N} \sum_{S_{j l} \in \mathcal{E}} \Delta t h^{d-1}\left|c_{j}^{n}-c_{l}^{n}\right| .
$$

We refer to CCL95b] for the proof of the estimate

$$
B V_{t} \leq C,
$$

where the constant depends on the data only, and we focus on the estimate of $B V_{x}$. 
For $i \in\{1, \ldots, d\}$, denote by $\mathcal{E}_{i}$ the set of interior edges orthogonal to the direction $\left\langle\mathbf{e}_{i}\right\rangle$, i.e., if $S_{j l} \in \mathcal{E}_{i}$, then either $l=j+\mathbf{e}_{i}$ or $l=j-\mathbf{e}_{i}$, and set

$$
B V_{x}=\sum_{n=0}^{N} \Delta t \sum_{i=1}^{d} B V_{i}^{n}, \quad B V_{i}^{n}:=\sum_{S_{j l} \in \mathcal{E}_{i}} h^{d-1}\left|c_{j}^{n}-c_{l}^{n}\right| .
$$

Note that the set of neighboring cells to a cell $T_{j}$ is given as $N(j)=\left\{j * \mathbf{e}_{i} \mid * \in\right.$ $\{+,-\}, i \in\{1, \ldots, d\}\}$. Furthermore, we have $F_{j, j \pm \mathbf{e}_{i}}(c)=F_{k, k \pm \mathbf{e}_{i}}(c)$, and $F_{j, j+\mathbf{e}_{i}}(c)$ $=-F_{k+\mathbf{e}_{i}, k}(c)$ for all $j, k \in \mathbb{Z}^{d}$.

If there is no boundary, i.e., $\mathcal{T}_{h}\left(\mathbb{R}^{d}\right)=\mathcal{T}_{h}(\Omega)$, then it has been proved in CH99a that $B V_{i}^{n+1} \leq B V_{i}^{n}$. This result uses the fact that the discrete solution is translation invariant under shifts $h \mathbf{e}_{i}, i=1, \ldots, d$, which yields

$$
\begin{aligned}
\left|c_{j}^{n+1}-c_{j \pm \mathbf{e}_{i} \mid}^{n+1}\right| \leq & \left(1-\sum_{\substack{* \in\{+,-\}, l \in\{1, \ldots, d\}}} Q_{j, j * \mathbf{e}_{l}}\right)\left|c_{j}^{n}-c_{j \pm \mathbf{e}_{i}}^{n}\right| \\
& +\sum_{\substack{* \in\{+,-\}, l \in\{1, \ldots, d\}}} Q_{j * \mathbf{e}_{l}, j \pm \mathbf{e}_{i} * \mathbf{e}_{l}\left|c_{j * \mathbf{e}_{l}}^{n}-c_{j \pm \mathbf{e}_{i} * \mathbf{e}_{l}}\right|,} \\
Q_{j l}:= & \frac{\Delta t}{h} \frac{F_{j l}\left(c_{j}\right)-F_{j l}\left(c_{l}\right)}{\left(c_{j}-c_{l}\right)}, \text { if } c_{j} \neq c_{l}, \text { and } Q_{j l}:=0 \text { else. }
\end{aligned}
$$

Note that the CFL condition (57) and the monotony of $\mathbf{F}_{j l}$ ensure that $0 \leq$ $Q_{j l}, \sum_{l \in N(j)} Q_{j l} \leq 1$. Let us now study the evolution of $B V_{i}^{n}$ for our bounded domain $\Omega$. Therefore, we define

$$
\overline{B V}_{i}^{n}:=B V_{i}^{n}+\sum_{S_{j, j \pm \mathbf{e}_{i}} \in \partial \mathcal{E}} h^{d-1}\left|c_{j}^{n}-\bar{c}_{j, j \pm \mathbf{e}_{i}}^{n}\right| .
$$

We first note that the the inequality (64) remains valid for any $S_{j, j \pm \mathbf{e}_{i}} \in \mathcal{E}_{i}$. Let us adapt (64) for a given edge $S_{j, j \pm \mathbf{e}_{i}} \in \partial \mathcal{E}$. The values $\bar{c}_{j, j \pm \mathbf{e}_{i}}^{n}$ are then given by (50) and (51). In addition, for $\mathbf{e}_{l} \neq \mathbf{e}_{i}$, define the values $c_{j \pm \mathbf{e}_{i}, j \pm \mathbf{e}_{i} \pm \mathbf{e}_{l}}^{n}$ through

$$
c_{j \pm \mathbf{e}_{i}, j \pm \mathbf{e}_{i} \pm \mathbf{e}_{l}}^{n}:= \begin{cases}\bar{c}_{j \pm \mathbf{e}_{i}, j \pm \mathbf{e}_{i} \pm \mathbf{e}_{l},}^{n} & \text { if } S_{j \pm \mathbf{e}_{l}, j \pm \mathbf{e}_{i} \pm \mathbf{e}_{l} \in \mathcal{E}}\left(\Rightarrow S_{j \pm \mathbf{e}_{i}, j \pm \mathbf{e}_{i} \pm \mathbf{e}_{l}} \in \partial \mathcal{E}\right), \\ \bar{c}_{j \pm \mathbf{e}_{l}, j \pm \mathbf{e}_{i} \pm \mathbf{e}_{l}}, & \text { if } S_{j \pm \mathbf{e}_{l}, j \pm \mathbf{e}_{i} \pm \mathbf{e}_{l}} \in \partial \mathcal{E} \\ \bar{c}_{j, j \pm \mathbf{e}_{l}}^{n}, & \text { else }\left(\Rightarrow S_{j, j \pm \mathbf{e}_{l}} \in \partial \mathcal{E}\right)\end{cases}
$$

From the definition of the scheme, we get

$$
c_{j}^{n+1}=c_{j}^{n}-\frac{\Delta t}{h} \sum_{\substack{* \in\{+,-\}, l \in\{1, \ldots, d\}}} F_{j, j * \mathbf{e}_{l}}\left(c_{j}^{n}\right)-F_{j, j * \mathbf{e}_{l}}\left(c_{j, j * \mathbf{e}_{l}}^{n}\right) .
$$

Furthermore, set

$$
\begin{aligned}
\delta_{j, j \pm \mathbf{e}_{i}}^{n} & :=c_{j, j \pm \mathbf{e}_{i}}^{n+1}-c_{j, j \pm \mathbf{e}_{i}}^{n} \\
& +\frac{\Delta t}{h} \sum_{\substack{* \in\{+,--\}, l \in\{1, \ldots, d\} \backslash\{i\}}} F_{j \pm \mathbf{e}_{i}, j \pm \mathbf{e}_{i} * \mathbf{e}_{l}}\left(\bar{c}_{j, j \pm \mathbf{e}_{i}}^{n}\right)-F_{j \pm \mathbf{e}_{i}, j \pm \mathbf{e}_{i} * \mathbf{e}_{l}}\left(c_{j \pm \mathbf{e}_{i}, j \pm \mathbf{e}_{i} \pm \mathbf{e}_{l}}^{n}\right) .
\end{aligned}
$$


We then have

$$
\begin{aligned}
c_{j}^{n+1} & -c_{j, j \pm \mathbf{e}_{i}}^{n+1}=c_{j}^{n}-c_{j, j \pm \mathbf{e}_{i}}^{n}-\frac{\Delta t}{h} \sum_{\substack{* \in\{+,-\}, l \in\{1, \ldots, d\}}} F_{j, j * \mathbf{e}_{l}}\left(c_{j}^{n}\right)-F_{j, j * \mathbf{e}_{l}}\left(c_{j, j * \mathbf{e}_{l}}^{n}\right) \\
& +\frac{\Delta t}{h} \sum_{\substack{* \in\{+,-\}, l \in\{1, \ldots, d\} \backslash\{i\}}}\left(F_{j \pm \mathbf{e}_{i}, j \pm \mathbf{e}_{i} * \mathbf{e}_{l}}\left(c_{j, j \pm \mathbf{e}_{i}}^{n}\right)-F_{j \pm \mathbf{e}_{i}, j \pm \mathbf{e}_{i} * \mathbf{e}_{l}}\left(c_{j \pm \mathbf{e}_{i}, j \pm \mathbf{e}_{i} * \mathbf{e}_{l}}^{n}\right)\right) \\
& -\delta_{j, j \pm \mathbf{e}_{i}}^{n} .
\end{aligned}
$$

Reordering these terms and using the shift invariance leads to the equivalent form (64)

$$
\begin{aligned}
\left|c_{j}^{n+1}-c_{j, j \pm \mathbf{e}_{i}}^{n+1}\right| & \leq\left(1-\sum_{\substack{* \in\{+,-\}, l \in\{1, \ldots, d\} \backslash\{i\}}} Q_{j, j * \mathbf{e}_{l}}-Q_{j, j \pm \mathbf{e}_{i}}\right)\left|c_{j}^{n}-c_{j, j \pm \mathbf{e}_{i}}^{n}\right| \\
& +Q_{j, j \mp \mathbf{e}_{i}}\left|c_{j}^{n}-c_{j, j \mp \mathbf{e}_{i}}^{n}\right|+\left|\delta_{j, j \pm \mathbf{e}_{i}}^{n}\right| \\
& +\sum_{\substack{* \in\{+,-\}, l \in\{1, \ldots, d\} \backslash\{i\}}} Q_{\left(j, j * \mathbf{e}_{l}\right),\left(j \pm \mathbf{e}_{i}, j \pm \mathbf{e}_{i} * \mathbf{e}_{l}\right)}\left|c_{j, j * \mathbf{e}_{l}}^{n}-c_{j \pm \mathbf{e}_{i}, j \pm \mathbf{e}_{i} * \mathbf{e}_{l}}^{n}\right| .
\end{aligned}
$$

Now, let us examine the process of compensation of terms when we sum the right hand side of (68) over $S_{j, j \pm \mathbf{e}_{i}} \in \partial \mathcal{E}$ and add it to the sum over $S_{j l} \in \mathcal{E}_{i}$ of the right hand side of (64). A careful examination leads to the estimate

$$
\overline{B V}_{i}^{n+1} \leq \overline{B V}_{i}^{n}+h^{d-1} \sum_{S_{j, j \pm \mathbf{e}_{i}} \in \partial \mathcal{E}}\left|\delta_{j, j \pm \mathbf{e}_{i}}^{n}\right| .
$$

Notice that whenever $F_{j \pm \mathbf{e}_{i}, j \pm \mathbf{e}_{i} * \mathbf{e}_{l}}\left(\bar{c}_{j, j \pm \mathbf{e}_{i}}^{n}\right)-F_{j \pm \mathbf{e}_{i}, j \pm \mathbf{e}_{i} * \mathbf{e}_{l}}\left(c_{j \pm \mathbf{e}_{i}, j \pm \mathbf{e}_{i} \pm \mathbf{e}_{l}}\right) \neq 0$ in (67), it amounts to a difference $F_{j, j * \mathbf{e}_{l}}\left(\bar{c}_{j, j \pm \mathbf{e}_{i}}^{n}\right)-F_{j, j * \mathbf{e}_{l}}\left(\bar{c}_{g h}^{n}\right)$ for some $S_{g h} \in \partial \mathcal{E}$ with $S_{j, j \pm \mathbf{e}_{i}} \cap S_{g h} \neq \emptyset$. Since the function $F_{j, j * \mathbf{e}_{l}}$ is $\mathcal{L}$-Lipschitz continuous, we have $\sum_{S_{j, j \pm \mathbf{e}_{i}} \in \partial \mathcal{E}}\left|\delta_{j, j \pm \mathbf{e}_{i}}^{n}\right| \leq \Delta_{t}^{n}+2(d-1) \mathcal{L} \frac{\Delta t}{h} \Delta_{x}^{n}$ with

$$
\Delta_{t}^{n}:=\sum_{S_{j l} \in \partial \mathcal{E}}\left|\bar{c}_{j l}^{n+1}-\bar{c}_{j l}^{n}\right|, \quad \Delta_{x}^{n}:=\sum_{S_{j l} \in \partial \mathcal{E}} \sum_{\substack{S_{g h} \in \partial \mathcal{E}, S_{j l} \cap S_{g h} \neq \emptyset}}\left|\bar{c}_{j l}^{n}-\bar{c}_{g h}^{n}\right| .
$$

By (57), we have $2(d-1) \mathcal{L} \frac{\Delta t}{h} \leq 1$. Let us estimate $\Delta_{x}^{n}$. We have $\Delta_{x}^{n}=\Delta_{x, \bullet}^{n}+$ $\sum_{i=1}^{n} \Delta_{x, i}^{n}$ with

$$
\begin{aligned}
\Delta_{x, \bullet}^{n} & :=\sum_{S_{j l} \in \partial \mathcal{E} \bullet} \sum_{\substack{S_{g h} \in \partial \mathcal{E}, S_{j l} \cap S_{g h} \neq \emptyset}}\left|\bar{c}_{j l}^{n}-\bar{c}_{g h}^{n}\right|, \\
\Delta_{x, i}^{n} & :=\sum_{S_{j l} \in \partial \mathcal{E}_{i}} \sum_{\substack{S_{g h} \in \partial \mathcal{E}, S_{j l} \cap S_{g h} \neq \emptyset}}\left|\bar{c}_{j l}^{n}-\bar{c}_{g h}^{n}\right| .
\end{aligned}
$$

Since $\bar{c}_{g h}^{n}=0$ for every $S_{g h} \in \partial \mathcal{E}_{\bullet}$, we have

$$
\Delta_{x, \bullet}^{n}=\sum_{S_{j l} \in \partial \mathcal{E}_{\bullet}} \sum_{\substack{S_{g h} \in \partial \mathcal{E} \backslash \partial \mathcal{E}_{\bullet}, S_{j l} \cap S_{g h} \neq \emptyset}}\left|\bar{c}_{g h}^{n}\right| .
$$


As each face $S_{j l}$ may have at most $2(d-1)$ neighboring faces, and by Lemma A.1 the cardinal of $\partial \mathcal{E}_{\bullet}$ is bounded by $\frac{C_{\#}}{h^{d-2}}$, we have

$$
\Delta_{x, \bullet}^{n} \leq 2(d-1) \frac{C_{\#}}{h^{d-2}}\|\bar{c}\|_{L^{\infty}(\partial \Omega \times(0, T))} .
$$

Similarly, we have, for $i \in\{1, \ldots, n\}$

$$
\sum_{S_{j l} \in \partial \mathcal{E}_{i}} \sum_{\substack{S_{g h} \in \partial \mathcal{E} \bullet, S_{j l} \cap S_{g h} \neq \emptyset}}\left|\bar{c}_{j l}^{n}-\bar{c}_{g h}^{n}\right| \leq 2(d-1) \frac{C_{\#}}{h^{d-2}}|| \bar{c} \|_{L^{\infty}(\partial \Omega \times(0, T))},
$$

and therefore $\Delta_{x, i}^{n} \leq \bar{\Delta}_{x, i}^{n}+\leq 2(d-1) \frac{C_{\#}}{h^{d-2}}\|\bar{c}\|_{L^{\infty}(\partial \Omega \times(0, T))}$ with

$$
\bar{\Delta}_{x, i}^{n}:=\sum_{S_{j l} \in \partial \mathcal{E}_{i}} \sum_{\substack{S_{g h} \in \partial \mathcal{E}_{i}, S_{j l} \cap S_{g h} \neq \emptyset}}\left|\bar{c}_{j l}^{n}-\bar{c}_{g h}^{n}\right| .
$$

Since $P_{i}$ is contractive, we have $\operatorname{diam}\left(P_{i}\left(S_{j l}\right) \cap P_{i}\left(S_{g h}\right)\right) \leq \operatorname{diam}\left(S_{j l}, S_{g h}\right) \leq 2 h$, if $S_{j l}, S_{g h} \in \partial \mathcal{E}_{i}, S_{j l} \cap S_{g h} \neq \emptyset$. Together with the nonoverlapping of the projections (Lemma A.2(2)) and the CFL condition (57), this shows that

$$
\sum_{t^{n} \in J} h \bar{\Delta}_{x, i}^{n} \leq C\|\bar{c}\|_{B V\left(A_{i} \times(0, T)\right)}
$$

if $\bar{c} \in B V\left(A_{i} \times(0, T)\right)$. Here and in the following, $C$ denotes a constant independent of $h$, and $\Delta t$. Similarly, we have

$$
\sum_{t^{n} \in J} h \Delta_{t}^{n} \leq C\|\bar{c}\|_{B V(\partial \Omega \times(0, T))} .
$$

Collecting the estimates, we deduce

$$
\overline{B V}_{i}^{n} \leq \overline{B V}_{i}^{0}+C \quad \forall t^{n} \in J .
$$

Let us bound $\overline{B V}_{i}^{0}$. We have $B V_{i}^{0} \leq C\left\|c_{0}\right\|_{B V(\Omega)} \leq C$ such that $(c f .(\underline{65})$ )

$$
\overline{B V}_{i}^{0} \leq C+\sum_{S_{j, j \pm \mathbf{e}_{i}} \in \partial \mathcal{E}} h^{d-1}\left|c_{j}^{0}-\bar{c}_{j, j \pm \mathbf{e}_{i}}^{0}\right| .
$$

Since the cardinal of $\partial \mathcal{E}$ is of order $\mathcal{O}\left(h^{d-1}\right)$ and since $\bar{c}$ and $c_{0}$ are bounded, we have $\sum_{S_{j, j \pm \mathbf{e}_{i} \in \partial \mathcal{E}}} h^{d-1}\left|c_{j}^{0}-\bar{c}_{j, j \pm \mathbf{e}_{i}}^{0}\right| \leq C$, and $\overline{B V}_{i}^{n} \leq C \forall t^{n} \in J$. All in all, we have $B V_{x} \leq C$. Together with (63), this completes the proof of the lemma.

Proof of the $B V$ estimate (7). Let $\varphi \in\left(\mathcal{C}_{c}^{\infty}(\Omega \times(0, T))\right)^{d}$ with $|\varphi(x, t)| \leq 1$ for all $(x, t) \in \Omega \times(0, T)$. Let $K_{x}$ and $K_{t}$ be some compact subsets of $\Omega$ and $(0, T)$, respectively, such that $\operatorname{supp}(\varphi) \subset K_{x} \times K_{t}$. Let $h$ be smaller than $\operatorname{dist}\left(K_{x}, \Omega^{c}\right) / 2 D_{d}$ such that $K_{x} \subset \Omega_{h}$. We have by Lemma A.4

$$
\begin{aligned}
\int_{\Omega \times \mathbb{R}^{+}} c \nabla \cdot \varphi \mathrm{dxdt} & =\int_{\Omega_{h}^{0}} \int_{0}^{T} c \nabla \cdot \varphi \mathrm{dxdt} \\
& =\int_{\Omega_{h}^{0}} \int_{0}^{T}\left(c-c_{h}\right) \nabla \cdot \varphi \mathrm{dxdt}+\int_{\Omega_{h}^{0}} \int_{0}^{T} c_{h} \nabla \cdot \varphi \mathrm{dxdt} \\
& \leq\|\nabla \cdot \varphi\|_{L^{\infty}(\Omega \times(0, T))}\left\|c-c_{h}\right\|_{L^{1}(\Omega \times(0, T))}+C_{B V} .
\end{aligned}
$$


At the limit $h \rightarrow 0$, by Lemma A.3 we get $\int_{\Omega \times \mathbb{R}^{+}} c \nabla \cdot \varphi \mathrm{dx} \mathrm{dt} \leq C$. This is true for an arbitrary function $\varphi \in\left(\mathcal{C}_{c}^{\infty}(\Omega \times(0, T))\right)^{d}$ with $|\varphi(x, t)| \leq 1$ for all $(x, t) \in \Omega \times(0, T)$. Therefore $\|c\|_{B V(\Omega \times(0, T))} \leq C_{B V}$.

\section{ACKNOWLEDGMENTS}

We would like to thank Jérôme Droniou, for precious advice and discussions. The first author would like to thank the Center for Scientific Computation and Mathematical Modeling, and the Department of Mathematics, University of Maryland, College Park, for the support of this work during his stay in the first half of 2003. The second author would like to thank the Abteilung für Angewandte Mathematik of Freiburg, and all its members for the very warm hospitality given during his stay in December 2002, where this work was initiated.

\section{REFERENCES}

[BCV95] S. Benharbit, A. Chalabi, and J.-P. Vila, Numerical viscosity and convergence of finite volume methods for conservation laws with boundary conditions, SIAM J. Numer. Anal. 32 (1995), no. 3, 775-796. MR1335655 (96c:65141)

[BLN79] C. Bardos, A. Y. LeRoux, and J.-C. Nédélec, First order quasilinear equations with boundary conditions, Comm. Partial Differential Equations 4 (1979), no. 9, 1017-1034. MR 0542510 (81b:35052)

[Car99] J. Carrillo, Entropy solutions for nonlinear degenerate problems, Arch. Ration. Mech. Anal. 147 (1999), no. 4, 269-361. MR.1709116 (2000m:35132)

[CCL95a] B. Cockburn, F. Coquel, and P. G. LeFloch, Convergence of the finite volume method for multidimensional conservation laws, SIAM J. Numer. Anal. 32 (1995), no. 3, 687-705. MR 1335651 (97f:65051)

[CCL95b] B. Cockburn, F. Coquel, and P.G. Lefloch, Convergence of the finite volumes method for multidimensional conservation laws, SIAM J. Num. Anal. 32 (1995), 687-705. MR 1335651 (97f:65051)

[CG95] B. Cockburn and H. Gau, A posteriori error estimates for general numerical methods for scalar conservation laws., Comput. Appl. Math. 14 (1995), 37-47. MR1339916

[CH99a] C. Chainais-Hillairet, Finite volume schemes for a nonlinear hyperbolic equation. Convergence towards the entropy solution and error estimate, M2AN Math. Model. Numer. Anal. 33 (1999), no. 1, 129-156. MR1685749 (2000a:65119)

[CHG01] C. Chainais-Hillairet and E. Grenier, Numerical boundary layers for hyperbolic systems in 1-D, M2AN Math. Model. Numer. Anal. 35 (2001), no. 1, 91-106. MR1811982 (2002m:65086)

[DIV03] J. Droniou, C. Imbert, and J. Vovelle, An error estimate for the parabolic approximation of multidimensional scalar conservation laws with boundary, Ann. Inst. H. Poincari Anal. Non Liniaire 21 (2004), no. 5, 689-714. MR2086755

[EGGH98] R. Eymard, T. Gallouët, M. Ghilani, and R. Herbin, Error estimates for the approximate solution of a nonlinear hyperbolic equation given by finite volume schemes, IMA Journal of Numerical Analysis 18 (1998), 563-594. MR1681074 (2000b:65180)

[EGH00] R. Eymard, T. Gallouët, and R. Herbin, Finite volume methods, Handbook of numerical analysis, Vol. VII (Amsterdam), North-Holland, Amsterdam, 2000, pp. 713-1020. MR 1804748 (2002e:65138)

[EGV03] R. Eymard, T. Gallouet, and J. Vovelle, Limit boundary conditions for fininte volume approximation of some physical problems, J. Comput. Appl. Math. 161 (2003), no. 2, 349-369. MR 2017019

[GM00] L. Gosse and C. Makridakis, Two a posteriori error estimates for one-dimensional scalar conservation laws, SIAM J. Numer. Anal. 38 (2000), no. 3, 964-988. MR1781211 (2002e:65129)

[HH02] R. Hartmann and P. Houston, Adaptive discontinuous Galerkin finite element methods for nonlinear hyperbolic conservation laws, SIAM J. Sci. Comput. 24 (2002), no. 3, 979-1004. MR1950521 (2004b:65149) 
[HMSW99] P. Houston, J.A. Mackenzie, E. Süli, and G. Warnecke, A posteriori error analysis for numerical approximation of Friedrichs system, Numer. Math. 82(3) (1999), 433-470. MR 1692130 (2000d:65163)

[IV03] C. Imbert and J. Vovelle, Kinetic formulation for multidimensional scalar conservation laws with boundary conditions and applications, SIAM J. Math. Anal. 36 (2004), no. 1, 214-232. MR2083859

[JS95] C. Johnson and A. Szepessy, Adaptive finite element methods for conservation laws based on a posteriori error estimates., Commun. Pure Appl. Math. 48 (1995), 199234. MR.1322810 (97b:76084)

[KKP02] S. Karni, A. Kurganov, and G. Petrova, A smoothness indicator for adaptive algorithms for hyperbolic systems, J. Comput. Phys. 178 (2002), no. 2, 323-341. MR 1899180 (2003c:65075)

[KNR95] D. Kröner, S. Noelle, and M. Rokyta, Convergence of higher order upwind finite volume schemes on unstructured grids for scalar conservation laws in several space dimensions, Numer. Math. 71 (1995), no. 4, 527-560. MR1355053(96j:65087)

[KO00] D. Kröner and M. Ohlberger, A-posteriori error estimates for upwind finite volume schemes for nonlinear conservation laws in multi dimensions, Math. Comput. 69 (2000), 25-39. MR 1659843 (2000i:65135)

[KO03] M. Küther and M. Ohlberger, Adaptive second order central schemes on unstructured staggered grids., Hyperbolic Problems: Theory, Numerics, Applications. Proceedings of the Ninth International Conference on Hyperbolic Problems held in Caltech, Pasadena, March 25-29, 2002 (Berlin/Heidelberg/New York) (T.Y. Hou and E. Tadmor, eds.), Springer, 2003. MR2053216

[Kru70] S. N. Kruzhkov, First order quasilinear equations with several independent variables., Mat. Sb. (N.S.) 81 (123) (1970), 228-255. MR0267257 (42:2159)

[Kuz76] N. N. Kuznetsov, The accuracy of certain approximate methods for the computation of weak solutions of a first order quasilinear equation, Ž. Vyčisl. Mat. i Mat. Fiz. 16 (1976), no. 6, 1489-1502, 1627. MR0483509 (58:3510)

[MNRR96] J. Málek, J. Nečas, M. Rokyta, and M. Ružička, Weak and measure-valued solutions to evolutionary PDEs, Chapman \& Hall, London, 1996. MR:1409366 (97g:35002)

[Ohl01a] M. Ohlberger, A posteriori error estimates for finite volume approximations to singularly perturbed nonlinear convection-diffusion equations, Numer. Math. 87(4) (2001), 737-761. MR1815733 (2001m:65120)

[Ohl01b] M. Ohlberger, A posteriori error estimates for vertex centered finite volume approximations of convection-diffusion-reaction equations, M2AN Math. Model. Numer. Anal. 35(2) (2001), 355-387. MR1825703 (2002a:65142)

[Ott96] F. Otto, Initial-boundary value problem for a scalar conservation law, C. R. Acad. Sci. Paris Sér. I Math. 322 (1996), no. 8, 729-734. MR 1387428 (97c:35132)

[Ser96] D. Serre, Systèmes de lois de conservation. II, Diderot Editeur, Paris, 1996, Structures géométriques, oscillation et problèmes mixtes. [Geometric structures, oscillation and mixed problems]. MR 1459989 (99e:35144)

[SH95] E. Süli and P.-A. Houston, Finite element methods for hyperbolic problems: a posteriori analysis and adaptivity, Report 96/09, Computing Laboratory, Oxford, May 1995; Inst. Math. Appl. Conf. Ser. New Ser., vol. 63, Oxford Univ. Press, New York, 1997, pp. 441-471. MR.1628356 (99i:65107)

[Sze91] A. Szepessy, Convergence of a streamline diffusion finite element method for scalar conservation laws with boundary conditions, RAIRO Modél. Math. Anal. Numér. 25 (1991), no. 6, 749-782. MR1135992 (92g:65115)

[Tad91] E. Tadmor, Local error estimates for discontinuous solutions of nonlinear hyperbolic equations., SIAM J. Numer. Anal. 28 (1991), 891-906. MR.1111445(92d:35190)

[Tan01] T. Tang, Error estimates of approximate solutions for nonlinear scalar conservation laws, Hyperbolic problems: theory, numerics, applications, Vol. I, II (Magdeburg, 2000) (Basel), Internat. Ser. Numer. Math., 140, vol. 141, Birkhäuser, Basel, 2001, pp. 873-882. MR:1871175

[Vig97] M. H. Vignal, Schemas Volumes Finis pour des equations elliptiques ou hyperboliques avec conditions aux limites, convergence et estimations d'erreur, Thesis, L'Ecole Normale Superieure, Lyon, 1997. 
[Vov02] J. Vovelle, Convergence of finite volume monotone schemes for scalar conservation laws on bounded domains, Num. Math. 90 (2002), no. 3, 563-596. MR.1884231 (2002k:65158)

Abteilung für Angewandte Mathematik, Universität Freiburg, Hermann-HerderStr.10, D-79104 Freiburg, Germany

E-mail address: mario@mathematik.uni-freibrug.de

Universite de Provence, CMi, F-13453 Marseille, France

E-mail address: vovelle@cmi.univ-mrs.fr 